\title{
NIR Responsive Tumor Vaccine in Situ for Photothermal Ablation and Chemotherapy to Trigger Robust Antitumor Immune Responses
}

\section{Lirong Zhang}

Department of Radiology,Affiliated Hospital of Jiangsu University

Jingjing Zhang

Jiangsu University

Lixia Xu

Jiangsu University

\section{Zijian Zhuang}

Department of Radiology,Affiliated Hospital of Jiangsu University

Jinajin Liu

Jiangsu University

Suwan Liu

Jiangsu University

Yunchao Wu

The Third People's Hospital of Changzhou

Aihua Gong

Jiangsu University

Miaomiao Zhang

Jiangsu University

Fengyi Du ( $\sim$ biodfy@ujs.edu.cn )

Nanjing Medical University Second Affiliated Hospital https://orcid.org/0000-0002-3555-421X

Research

Keywords: Polydopamine nanoparticle, Hyaluronic acid, Photothermal therapy, Immune therapy

Posted Date: February 11th, 2021

DOl: https://doi.org/10.21203/rs.3.rs-175988/v1

License: (c) (1) This work is licensed under a Creative Commons Attribution 4.0 International License.

Read Full License 


\section{Abstract}

Background: Therapeutic tumor vaccine (TTV) that induces tumor-specific immunity has enormous potentials in tumor treatment, but high heterogeneity and poor immunogenicity of tumor seriously impaires its clinical efficacy. Herein, a novel NIR responsive tumor vaccine in situ (HA-PDA@IQ/DOX HG) was prepared by integrating hyaluronic acid functionalized polydopamine nanoparticles (HA-PDA NPs) with immune adjuvants (Imiquimod, IQ) and doxorubicin (DOX) into thermal-sensitive hydrogel.

Results: HA-PDA@IQ NPs with high photothermal conversion efficiency (41.2\%) and $T_{1}$-relaxation efficiency were using HA as stabilizer by the one-pot oxidative polymerization. Then, HA-PDA@IQ Ioaded DOX via $\pi-\pi$ stacking and mixed with thermal-sensitive hydrogel to form the HA-PDA@IQ/DOX HG. The hydrogel-confined delivery mode endowed HA-PDA@IQ/DOX NPs with multiple photothermal ablation performance once injection upon NIR irradiation due to the prolonged retention in tumor site. More importantly, this mode enabled HA-PDA@IQ/DOX NPs to promote the DC maturation, memory T cells in lymphatic node as well as cytotoxic T lymphocytes in spleen.

Conclusion: Taken together, the HA-PDA@IQ/DOX HG could be served as a theranostic tumor vaccine for complete photothermal ablation to trigger robust antitumor immune responses.

\section{Introduction}

Therapeutic cancer vaccine (TCV) that aims to stimulate tumor antigen-specific immunological responses is emerging as promising technology in cancer immunotherapy in recent years [1-3]. In particular, substantial tumor antigen-based tumor vaccines have been deeply explored in basic research and clinic trials, resulting in the approval of the prostatic acid phosphatase-based peptide vaccination by the U.S. Food and Drug Administration [4-6]. Despite great progress, the therapeutic effect of TCV is impaired due to the complex physiological characteristics of tumor, including high heterogeneity with various somatic mutations and corresponding neoantigens among patients [7-9]. Thus, it is significant to overcome these hurdles to eliminate and inhibit cancer.

Personalized TCV in situ represents a promising strategy to overcome tumor heterogeneity by inducing autologous whole tumor antigens release [10-12]. Up to now, a variety of immuno-stimulatory treatments for development of TCV, such as oncolytic viruses [13, 14], radiotherapy $[15,16]$, chemotherapy [17] and phototherapy $[18,19]$, have been reported to generate tumor-associated antigens in situ by inducing immunogenic cell death (ICD) [20-22]. Among these treatments, near infrared (NIR) laser mediated photothermal ablation (PTA) has been regarded as a powerful technique due to its high selectivity and minimal side effects $[23,24]$. Its therapeutic effects required both the photosensitizer accumulation at the tumor site and localized NIR laser exposure, leading to the heating temperature up to $50{ }^{\circ} \mathrm{C}$ to realize tumor coagulative necrosis $[25,26]$. However, the ineffectivity of PPT-mediated antitumor response which led to recurrence and metastasis suggested that additional therapeutic interventions may be needed to completely eradicate tumor $[27,28]$. 
Mounting evidences have demonstrated that simulation of dendritic cells (DCs) with immunoadjuvants could promote the antigens presentation and immune response to relieve immunosuppression $[29,30]$. Mature DCs capture antigens derived from cancer and assembled with the major histocompatibility complex (MHC) to trigger robust $\mathrm{CD} 8^{+}$cytotoxic $T$ lymphocyte $(\mathrm{CTL})$ response [31, 32]. For instance, imiquimod (R837) as a potent TLR7 agonist has been integrated into distinct TCV nanosystem to collaborate with radiotherapy [33, 34], photothermal therapy [35], photodynamic therapy (PDT) [36, 37], and chemotherapy [38]. However, the actual therapeutic effects of these TCV were impaired due to their complicated manufacture process and unsatisfactory performance.

In order to address above limitations, NIR responsive cancer vaccine in situ (HA-PDA@IQ/DOX HG) was fabricated to stimulate strong antitumor immunity. Briefly, HA and IQ were integrated into polydopamine nanoparticles (PDA NPs) using potassium permanganate mediated-one step polymerization. A certain amount of DOX were adsorbed on the surface of PDA NPs through the interaction of $\pi-\pi$ accumulation and electrostatic attraction. Then, the chemical and physical properties of HA-PDA@IQ/DOX NPs were characterized and its photothermal conversion efficiency was calculated. The ability of HA-PDA@IQ/DOX NPs to induce dendritic cells (DCs) maturation was studied using bone morrow derived dendritic cells (BMDCs) in vitro. Furthermore, thermosensitive hydrogel loaded HA-PDA@IQ/DOX NPs was injected in situ into tumor site to investigate the influence on tumor growth and immune system. After NIR irradiation, we focused on the effect of DC maturation and memory T cells in lymphatic node as well as cytotoxic T lymphocytes in spleen.

\section{Results}

\section{Preparation and characterization of HA-PDA@IQ/DOX NPs}

As shown in Scheme1, HA-PDA@IQ NPs were synthesized by the one-pot self-polymerization of dopamine initiated by $\mathrm{KMnO}_{4}$ in the presence of stabilizer (HA) and immune adjuvant (IQ). Then, the chemotherapy drug (DOX) was loaded via $\pi-\pi$ stacking to form HA-PDA@IQ/DOX NPs. As shown in Figure 1a, the TEM result showed that the prepared HA-PDA@IQ/DOX NPs were spherical, uniformly distributed without obvious aggregation. The average size and zeta potentials of HA-PDA@IQ NPs and HA-PDA@IQ/DOX NPs were 136.4 nm and 150.9 nm,-23.1 mV and -18.8 mV, respectively (Figure 1b, c). The changes in size and zeta potential were attributed to DOX loading. In addition, elemental mapping images displayed that Mn element has been successfully integrated into HA-PDA@IQ/DOX NPs during oxidative polymerization (Figure 1d). XRD spectrum indicated that there was broad diffraction peak at 23 ', which represented non crystalline phase structure in HA-PDA@IQ/DOX NPs (Figure 1e). As shown in Figure 1f, the chemical structure and composition of HA-PDA@IQ/DOX NPs were analyzed by X-ray photoelectron spectroscopy (XPS). There were four peaks at $641.48 \mathrm{eV}, 532.59 \mathrm{eV}, 399.88 \mathrm{eV}$ and 296.29 $\mathrm{eV}$, corresponding to the four elements of manganese, oxygen, nitrogen and carbon, which proved the element composition of HA-PDA@IQ/DOX NPs. As shown in Figure 1g, there were two typical bindingenergy peaks at $654.2 \mathrm{eV}$ and $641.1 \mathrm{eV}$, corresponding to $\mathrm{Mn}(\mathrm{IV})_{2 \mathrm{p} 1 / 2}$ and $\mathrm{Mn}(\mathrm{IV})_{2 \mathrm{p} 3 / 2}$, respectively, which further confirmed the presence of Mn element in HA-PDA@IQ/DOX NPs. The C1s spectrum showed 
three main peaks, $287.7 \mathrm{eV}, 286.2 \mathrm{eV}$, and $284.4 \mathrm{eV}$ (Figure $1 \mathrm{~h}$ ), corresponding to $\mathrm{C}=0, \mathrm{C}-\mathrm{N}$ and $\mathrm{C}-\mathrm{C}$, respectively. The 01 spectrum had two obvious peaks at $532.98 \mathrm{eV}$ and $531.1 \mathrm{eV}$, which were attributed to $\mathrm{C}=0$ and $\mathrm{C}-\mathrm{O}$ (Figure 1i). The N1s spectrum showed four peaks, $399.9 \mathrm{eV}, 399.5 \mathrm{eV}, 398.9 \mathrm{ev}$ and $400.5 \mathrm{eV}$, which indicated the presence of $\mathrm{N}$ atoms (Figure $1 \mathrm{j}$ ). These hydrophilic chemical groups endowed the HAPDA@IQ/DOX NPs with superior dispersibility and stability in aqueous solution, which were essential for biomedical applications.

\section{Photothermal properties of HA-PDA@IQ/DOX NPs}

Photothermal conversion performance of HA-PDA@IQ/DOX NPs was investigated upon $808 \mathrm{~nm}$ laser irradiation (Figure 2a).Firstly, the UV-Vis spectra of HA-PDA@IQ/DOX NPs showed a wide absorption band extending to the infrared region, where the absorption intensity increased with the increase of concentration $(0.2-1.0 \mathrm{mg} / \mathrm{mL})$ in Figure $\mathbf{2 b}$. Next, we studied the temperature changes of HAPDA@IQ/DOX NPs solution with different concentrations under laser irradiation of $2 \mathrm{~W} / \mathrm{cm}^{2}$, as shown in Figure 2c. Generally, the temperature of each group from 0.1 to $1 \mathrm{mg} / \mathrm{mL}$ quickly increased with the increase of HA-PDA@IQ/DOX NPs concentration. By contrast, there was no obvious change in temperature in de-ionized water group. Notably, the temperature of HA-PDA@IQ/DOX NPs group even reached $59.4{ }^{\circ} \mathrm{C}$ within 5 minutes under $808 \mathrm{~nm}$ laser irradiation when the concentration was $1 \mathrm{mg} / \mathrm{mL}$. In addition, we tested the temperature changes of HA-PDA@IQ/DOX NPs $(1 \mathrm{mg} / \mathrm{mL})$ group at different power densities from 1 to $2.5 \mathrm{~W} / \mathrm{cm}^{2}$ (Figure 2d), and recorded the heat map using an infrared camera (Figure 2e). As we expected, the temperature exhibited power density-dependent manner, even exceeded $60^{\circ} \mathrm{C}$ under the power density of $2.5 \mathrm{~W} / \mathrm{cm}^{2}$. Compared with commercial photosensitizer ICG, photothermal stability of HA-PDA@IQ/DOX NPs $(200 \mu \mathrm{L}, 1.0 \mathrm{mg} / \mathrm{mL})$ was studied during the five cycles of on/off laser. As shown in Figure $\mathbf{2 f}$, the temperature of the ICG solution gradually decreased after five rounds of repeated laser irradiation due to inherent photoquenching. However, the temperature of the HAPDA@IQ/DOX NPs solution remained stable during five irradiation cycles, indicating that HAPDA@IQ/DOX NPs have a highly stable photothermal effect. Besides, there was no significant change in the Vis-NIR absorbance of HA-PDA@IQ/DOX NPs before and after five rounds of repeated laser irradiation (Figure $\mathbf{2} \mathbf{g}$ ). According to the calculation formula in Figure $\mathbf{2} \mathbf{h}$ and $\mathbf{2} \mathbf{i}$, the photothermal conversion efficiency ( $\eta$ ) of HA-PDA@IQ NPs was 41.2\%, which was obviously much higher than those of previously reported PTT agents [39]. The high $\eta$ of HA-PDA@IQ/DOX NPs could be attributed to its strong absorption at the near-infrared (NIR) wavelength of $808 \mathrm{~nm}$ and the increase in electron transport efficiency induced by manganese element.

\section{Dox loading and release}

It is well known that aromatic molecules can be efficiently loaded on nanoparticles with delocalized $\pi$ electrons through $\pi-\pi$ stacking and hydrophobic interaction. Since PDA contains a delocalized $\pi$ electronic structure, we envisioned that HA-PDA@IQ can also be used as a nano-drug delivery carrier to load the doxorubicin (common chemotherapy drug, DOX). In our experiment, HA-PDA@IQ and DOX were mixed in different ratios and stirred overnight at room temperature. After washing, the obtained HA- 
PDA@IQ/DOX NPs were detect by UV-Vis-NIR spectroscopy (Figure 3a). Compared with pure HA-PDA@IQ NPs, the HA-PDA@IQ/DOX NPs has a characteristic absorption peak at $490 \mathrm{~nm}$, indicating that DOX was successfully loaded. As the weight ratio of DOX: PDA increased, the drug loading on the nanoparticles also increased (Figure $3 b$ ). The maximum loading reached about 130\% (DOX: PDA, w/w), which seemed to be much higher than traditional polymer-based NDDS, and the loading capacity at this time reached $37.2 \%$.

Next, we studied the drug release behavior of DOX in HA-PDA@IQ/DOX NPs.The supernatant was collected by dissolving HA-PDA@IQ/DOX in PBS with different pH values (5.0 and 7.4) with gentle agitation (Figure 3c). The amount of released DOX was analyzed by UV-Vis-NIR spectroscopy. After incubation of 24 hours, the released $\mathrm{DOX}$ at pH 5.0 was about $45 \%$, while the released $\mathrm{DOX}$ at pH 7.4 was only $15.2 \%$. Therefore, DOX release seems to be more pronounced at a lower $\mathrm{pH}$ (5.0) compared with physiological $\mathrm{pH}$ (7.4). This acid-triggered release was attributed to the protonation of the amino group in the DOX molecule under low $\mathrm{pH}$. Then, we wondered whether the NIR-induced heating could promote the release of DOX. The HA-PDA@IQ/DOX NPs in pH 5.0 and 7.4 PBS were irradiated by $808 \mathrm{~nm}$ laser (2 $\mathrm{W} / \mathrm{cm}^{2}, 5$ minutes) at different time points. As shown in Figure $3 \mathbf{d}$, the cumulative release of DOX in HAPDA@IQ/DOX NPs was measured by UV-Vis-NIR spectroscopy. Compared with no laser irradiation treatment, released DOX exhibited periodical fluctuation along laser on/off irradiation, demonstrating that irradiation could significantly enhanced the release of DOX from HA-PDA@IQ/DOX NPs. In view of these features, acidic environment of tumor tissue and HA-PDA@IQ/DOX NPs-mediated photothermal effect were conducive to the controllable release of DOX.

\section{Biocompatibility and PTA effect of HA-PDA@IQ NPs in vitro}

In order to eliminate the interference of DOX, HA-PDA@IQ NPs without DOX was used in the following experiments. To evaluate the biocompatibility, the CCK-8 experiment was performed using two types of cells, including normal mouse embryonic fibroblast (MEF) cells and mouse breast cancer cell 4T1 cells. Both MEF cells and 4T1 cells were cultured with different concentrations of HA-PDA@IQ NPs $(0,200,400$, $600,800,1000,1200 \mu \mathrm{g} / \mathrm{mL}$ ) for 48 h, respectively. As shown in Figure 5a, HA-PDA@IQ NPs showed negligible cytotoxicity to both MEF cells and 4T1 cells. Even at a high concentration of $1200 \mu \mathrm{g} / \mathrm{mL}$, both these cell survival rate exceeded $80 \%$. The hemolysis test was also used to evaluate the blood compatibility of HA-PDA@IQ NPs. As shown in Figure 5d, compared with the deionized water group, the absorbance at $541 \mathrm{~nm}$ did not exceed 0.04 even at $1200 \mu \mathrm{g} / \mathrm{mL}$. These above results indicated that the prepared HA-PDA@IQ NPs has good biocompatibility.

After that, we evaluated the effect of HA-PDA@IQ NPs mediated PTA on tumor growth in vitro. The tumor cells 4T1 and normal cell MEF were co-cultured with different concentrations of HA-PDA@IQ NPs and irradiated with $808 \mathrm{~nm}$ laser for 5 minutes $\left(2 \mathrm{~W} / \mathrm{cm}^{2}\right)$. It can be seen from Figures $4 \mathrm{~b}$ and $4 \mathbf{c}$ that the cell viability of $4 \mathrm{~T} 1$ cells was lower than $50 \%$ at $800 \mu \mathrm{g} / \mathrm{mL}$, while the cell viability of MEF cells was higher than $80 \%$ even at same concentration. The findings proved that PTA significantly inhibited the tumor cell viability, but has little effect on normal cells. The possible reason was that normal cells are more resistant 
to temperature than cancer cells. Furthermore, live/dead cell assay was conducted to verify the effect of PTA on tumor cells. It can be seen from Figure $4 \mathbf{e}$ that there was almost no cell death in control, NIR and HA-PDA@IQ NPs treatment. In contrast, HA-PDA@IQ NPs+NIR caused mass cell death, indicating that HAPDA@IQ NPs had promising potential in PTA of tumor.

\section{The effect of HA-PDA@IQ NPs on DC maturation.}

Firstly, the intracellular localization and real-time cell uptake of HA-PDA@IQ/DOX NPs were studied via fluorescence microscopy after incubation 4T1 cells with HA-PDA@IQ/DOX NPs for 24 hours. As shown in Figure 5a, red fluorescence could be observed in the cytoplasm, but no signal in nucleus. These findings showed that HA-PDA@IQ/DOX NPs could be phagocytosed into the cytoplasm and distributed around nucleus.

During the antitumor immune response, DC as a type of antigen presenting cell (APC) plays an essential role in capturing the neoantigen and activating cytotoxicity $T$ lymphocyte. Therefore, immature bone marrow-derived dendritic cells (BMDC) were used to evaluate the effect of HA-PDA@IQ NPs on DC maturation. Flow cytometry analysis was used to study the expression of CD80 and CD86 on the surface of BMDCs, which represented the level of DC maturation. Figure5b showed the expression of CD80 and CD86 on the surface of BMDCs increased with the increase of HA-PDA@IQ NPs concentration. Compared with PBS treatment (only 2.33\%), CD80 and CD86 expression in HA-PDA@IQ NPs treatment was up to $10.31 \%$. Based on these findings, we have reason to believe that resultant HA-PDA@IQ NPs have a powerful effect on triggering DC maturation, which may be beneficial to anti-tumor immune activation.

\section{MRI performance of HA-PDA@IQ/DOX NPs in Vitro and in Vivo.}

Due to the Mn element doping, we speculated that HA-PDA@IQ/DOX NPs might possess the capability to be utilized as a potential contrast agent (CAs) for MR imaging. Herein, the 3T MR scanner was used to study the $T_{1}$-weighted MR of HA-PDA@IQ/DOX NPs in vitro. As shown in Figure 7a, there was a positive correlation between HA-PDA@IQ/DOX NPs solutions with different Mn concentrations (0-0.2 nM) and $T_{1^{-}}$ weighted MR signal intensity. Furthermore, HA-PDA@IQ/ DOX NPs encapsulated with F127 hydrogel was in situ injected into tumor site to access their imaging capability. As shown in Figure $7 \mathbf{b}$, strong signal could be observed in tumor region after HA-PDA@IQ/DOX NPs injection. Then, we further studied the retention and distribution of HA-PDA@IQ/DOX NPs at tumor site for 48 hours. As shown in Figure 7c, single HA-PDA@IQ/ DOX NPs disappeared quickly within 6 hours, while HA-PDA@IQ/DOX HG still well remained in tumor region under 48 hours. It could be seen that HA-PDA@IQ/DOX HG had a strong retention effect at tumor site, which was conducive to the recruitment of APCs as well as recognition of neoantigen derived from necrotic tumor cells.

\section{Immune responses assessment in vivo}

In view of the excellent photothermal performance and biocompatibility, we further studied the immunoregulatory function of HA-PDA@IQ/DOX NPs in vivo using 4T1 subcutaneous tumor-bearing 
mice as animal model (Figure 7a). When the average tumor volume reached about $100 \mathrm{~mm}^{3}$, the 4T1 tumor-bearing mice were divided into five groups (PBS, HA-PDA NPs, HA-PDA@IQ NPs, HA-PDA@IQ/DOX NPs and HA-PDA@IQ/DOX HG) for in-situ injection under the $808 \mathrm{~nm}$ NIR irradiation (2 W/ $\left./ \mathrm{cm}^{2}, 5 \mathrm{~min}\right)$. The maturation of DC cells in the inguinal lymph nodes of mice with breast cancer was detected by flow cytometry (Figure $7 \mathrm{~b})$. The data showed that the ratio of $\operatorname{CD} 11 \mathrm{c}^{+} / \mathrm{CD} 80^{+}(1.27 \%)$ and $\mathrm{CD} 11 \mathrm{c}^{+} / \mathrm{CD} 86^{+}$cells (1.52\%) in HA-PDA treatment group was a little bit higher than that in control group PBS $(1.03 \%, 1.46 \%)$, which may be attributed to photothermal treatment influence. Notably, the proportion of $\mathrm{CD} 11 \mathrm{c}^{+} / \mathrm{CD} 80^{+}$ cells and the proportion of $\mathrm{CD} 11 \mathrm{c}^{+} / \mathrm{CD} 86^{+}$cells in HA-PDA@IQ NPs treatment group were higher than those in HA-PDA NPs treatment group, confirming that immune adjuvant IQ doping could stimulate DC maturation. Meanwhile, the ratio of $\mathrm{CD} 11 \mathrm{c}^{+} / \mathrm{CD} 80^{+}$and $\mathrm{CD} 11 \mathrm{c}^{+} / \mathrm{CD} 86^{+}$cells in HA-PDA@IQ/DOX HG group were higher than that in HA-PDA@IQ/DOX NPs group. In the HA-PDA@IQ/DOX HG group, the DC maturation rate was the highest $\left(6.43 \%\right.$ for $\mathrm{CD} 11 \mathrm{c}^{+} / \mathrm{CD} 80^{+}$cells and $6.67 \%$ for $\mathrm{CD} 11 \mathrm{c}^{+} / \mathrm{CD} 86^{+}$cells). The reason may be that the addition of the gel prolonged the residence time of the nanoparticles in the tumor and enhanced the recruitment of APC cells. In order to study the potential utility of this treatment strategy for the long-term prevention of tumor recurrence, we used flow cytometry to detect the inducibility of effector $\mathrm{T}$ cells in inguinal lymph nodes ( $\left.\mathrm{CD} 3^{+} / \mathrm{CD} 8^{+} / \mathrm{CD} 44^{\text {high }} / \mathrm{CD} 62 \mathrm{~L}^{\text {low }}\right)$. As shown in figure $7 \mathrm{c}$, the ratio of memory T cells reached 22.64\% under HA-PDA@IQ/DOX HG treatment, which was 6.2 times compared with control group (PBS). These results demonstrated that HA-PDA@IQ/DOX HG had the ability to promote memory $\mathrm{T}$ cells production.

Cytotoxic $\mathrm{T}$ lymphocytes (called CTL or $\mathrm{CD} 8^{+} \mathrm{T}$ cells) are essential for the anti-tumor immune response where they are activated by tumor-derived antigens and directly destroy target cells. In this experiment, each group of splenocytes was co-stained with anti-CD3 and anti-CD8a antibodies and was measured by flow cytometry. As shown in Figure 7d, the percentage of $\mathrm{CD}^{+} / \mathrm{CD} 8^{+} \mathrm{T}$ cells in PBS, HA-PDA NPs, HAPDA@IQ NPs, HA-PDA@IQ/DOX NPs and HA-PDA@IQ/DOX HG group were 2.30\%, 2.68\%, 5.05\%, 5.43\% and $5.68 \%$, respectively. It was worth noting that the percentage of $\mathrm{CD}^{+} / \mathrm{CD} 8^{+} \mathrm{T}$ cells in HA-PDA@IQ NPs (5.05\%) group was about 1.88 times as that in HA-PDA NPs (2.68\%). This enhancement of CTL might be ascribed to the introduction of IQ, which remarkably stimulated the DC cells maturation and enhanced the tumor-derived antigens presentation function.

\section{The antitumor effect of HA-PDA@IQ/DOX HG in vivo}

To verify the therapeutic effect of HA-PDA@IQ/DOX HG, subcutaneous 4T1-Luc breast tumor model was established in the inner mammary gland of lower left posterior breast (Figure 8a). After 7 days of treatment, the tumors were remarkably inhibited in HA-PDA@IQ/DOX HG group compared with other groups in figure $\mathbf{8 b}$. It was noted that the tumors in HA-PDA@IQ/DOX HG group completely disappeared on the $20^{\text {th }}$ day. Individual tumor growth curves of each group further confirmed this trend (Figure $\mathbf{8 c}$ ). It can be seen that HA-PDA@IQ/DOX HG with irradiation possessed application potential in eliminating the tumor in situ. 


\section{The PTA performance of HA-PDA@IQ/DOX HG in vivo}

In order to evaluate the photothermal performance in vivo, digital NIR thermal imager was chosen to realtime monitor temperature profile of tumor site after in situ injection of HA-PDA@ IQ/DOX HG. As shown in Figure $9 a$ and $9 b$, the surface temperature of the tumor in each group increased sharply, except for the PBS group. Notably, the temperature of tumor in HA-PDA@IQ/DOX HG group reached its maximum and even up to $56.6^{\circ} \mathrm{C}$ after 5 min of laser irradiation. After that, irradiation was performed every other day for a total of three times and corresponding tumor volume was recorded at certain time interval. As shown in Figure 9c, the growth curves of the tumor within 20 days further verified that HA-PDA@IQ/DOX HG group exhibited optimal anti-tumor effect compared to other control groups. However, there was no significant change in body weight among all groups in Figure $9 \mathrm{~d}$. It was worth noting that the survival period of the mice in HA-PDA@IQ/DOX HG group was as long as 60 days, while the average life span of mice in the control group was shorter than 30 days in Figure 9 e. The life span of mice in other treatment groups was extended to varying degrees, which was attributed to the lack of DOX and hydrogel collaboration. These results proved that PTA, hydrogel and chemotherapy could reinforce each other, finally obtain an ideal therapeutic effect. In addition, in order to verify its biological safety in vivo, the heart, liver, spleen, lung and kidney of the mice were taken out for H\&E staining analysis (Figure 9 f). The images showed that there were no inflammatory lesions or obvious tissue damage.

\section{Conclusions}

In this study, we reported that a novel NIR responsive nanovaccine (HA-PDA@IQ/DOX HG) for in situ PTA and chemotherapy can boost anti-tumor immunity. During the synthetic process, HA was used as template molecule to encapsulate IQ and assist dopamine oxidative polymerization catalyzed by potassium permanganate. In order to enhance the PTA-mediated ICD, the chemotherapeutic drug DOX was efficiently attached by HA-PDA@IQ NPs through $\pi-\pi$ accumulation and electrostatic interaction. Upon NIR irradiation, the resultant HA-PDA@IQ/DOX NPs not only exhibited highly photothermal conversion efficiency of $41.2 \%$, but also accelerated DOX release. In addition, HA-PDA@IQ/DOX NPs as CA were capable of MR imaging performance due to the introduction of Mn doping. Furthermore, HAPDA@IQ/DOX NPs with favorable biocompatibility remarkably stimulated DC maturation in vitro. Most importantly, HA-PDA@IQ/DOX NPs loaded into thermosensitive hydrogel not only enable the repeated PTA, but also potentiated the antitumor immune responses. These results indicated that that HAPDA@IQ/DOX HG possessed a promising potential to amplify antitumor immunotherapeutic efficacy.

\section{Experimental Section}

\section{Materials}

Dopamine (DA), Hyaluronic acid (HA), Imiquimod (IQ) and Doxorubicin (DOX) were purchased from Aladdin Reagent Company (Shanghai, People's Republic of China). Cell counting KIT-8 (CCK-8) and 4', 6diamidino-2-phenylindole (DAPI) were obtained from Thermo Fisher Scientific. Phosphate buffered saline 
(PBS) was purchased from Hyclone (Logan City, Utah, USA). Dulbecco's Minimum Essential Medium (DMEM), RPMI Medium Modified (RPMI-1640) and Fetal bovine serum (FBS) were procured from Hyclone (Logan, UT, USA). Trypsin was obtained from Sigma-Aldrich Company (USA). Fluorescein diacetate (FDA) and Propidium iodide (PI) were purchased from Sigma (New York, NY, USA). De-ionized water was used in the experiments.

\section{Synthesis of HA-PDA@IQ/DOX NPs}

$0.1 \mathrm{~g}$ hyaluronic acid (HA) was dissolved in $200 \mathrm{~mL}$ deionized water, which was stirred continuously at room temperature until dissolved. Then, $0.1 \mathrm{~g}$ Dopamine (DA), $0.01 \mathrm{~g}$ Imiquimod (IQ) and $200 \mu \mathrm{L} \mathrm{KMnO}{ }_{4}$ $(3 \mathrm{mg} / \mathrm{mL}$ ) were slowly added into the HA solution under continuous stirring for $2 \mathrm{~h}$. The colorless solution immediately turned light yellow and gradually turned dark brown. Afterwards, the reaction solution was centrifuged at $3000 \mathrm{rpm}$ for $10 \mathrm{~min}$ to remove the sediment. The supernatant was poured into a cut-off dialysis bag (MWCO 10000) for dialysis for 5 days, changing the water every 6 hours. The obtained dialysate was freeze-dried in a vacuum freeze dryer (Boyikang, Beijing, China) for 3 days to obtain a black powder (HA-PDA@IQ NPs). Finally, $1 \mathrm{mg} / \mathrm{mL}$ doxorubicin was added into $1 \mathrm{mg} / \mathrm{mL}$ HAPDA@IQ NPs solution and stirred at room temperature for 24 hours, then centrifuged at 10, $000 \mathrm{rpm}$ for 20 minutes and collect the precipitate. The obtained precipitate was freeze-dried in a vacuum freeze dryer for 1 day to obtain HA-PDA@IQ/DOX NPs.

\section{Instruments and characterizations of HA-PDA@IQ NPs}

The phase structure was analyzed by X-ray diffraction (XRD) on Rigaku smartlab 9kw (Rigaku, Japan), equipped with a $\mathrm{Cu}$ Ka radiation source $(\lambda=0.15418 \mathrm{~nm})$, with a slit of $0.5^{\circ}$ and a scanning speed of 7 $\%$ min. The X-ray photoelectron spectroscopy (XPS) was performed on Thermo Fisher Nexsa (America). UV-Vis absorption was identified by A560 dual-beam UV-Vis spectrophotometer (AOE Instruments, Shanghai). The morphology and the elemental mapping images of the HA-PDA@IQ/DOX were observed by transmission electron microscopy (TEM, FEI Talos F200S, America).

\section{Photothermal conversion efficiency}

In order to measure the photothermal conversion efficiency ( $\eta$ ), $200 \mu \mathrm{L}$ of HA-PDA@IQ NPs aqueous solution $(1 \mathrm{mg} / \mathrm{mL})$ in the EP tube was irradiated using an $808 \mathrm{~nm}$ NIR laser with a power density of 2.0 $\mathrm{W} / \mathrm{cm}^{2}$ for $600 \mathrm{~s}$ and then turn off the laser lasted $600 \mathrm{~s}$. An infrared camera was used to monitor the temperature change of the solution for $1200 \mathrm{~s}$. The $\eta$ value of the sample was determined by the following formula[40, 41]: 


$$
\begin{aligned}
& \eta=\frac{\mathrm{hS}\left(\Delta T_{\max , \operatorname{mix}}-\Delta T_{\max H_{2} O}\right)}{I\left(1-10^{-A 808}\right)} \\
& \mathrm{hS}=-\frac{\sum_{i} m_{i} C_{p, i}}{\mathrm{t}} \ln \theta \\
& \theta=\frac{\Delta T}{\Delta T \max , \operatorname{mix}}
\end{aligned}
$$

In the equations, $\mathrm{h}$ is the heat transfer coefficient; $\mathrm{S}$ is the surface area of the container; $\Delta T_{\text {max,mix }}$ is the temperature change of the HA-PDA@IQ/DOX NPs dispersion at the maximum steady-state temperature. $\Delta T_{\max , \mathrm{H} 2 \mathrm{O}}$ is the temperature change of water at the maximum steady-state temperature. / is the laser power, $A_{808}$ is the absorbance of HA-PDA@IQ/DOX NPs at the wavelength of $808 \mathrm{~nm}$ in aqueous solution. Compared with solvent (water, $2 \times 10^{-3} \mathrm{Kg}$ ), mass of NPs $\left(2 \times 10^{-8} \mathrm{Kg}\right)$ was too little. Generally, the specific heat of water is much higher than other materials. Consequently, the $\mathrm{m}_{\mathrm{NPs}}$ and $C_{\mathrm{p}, \mathrm{NPs}}$ of NPs were neglected. $m_{\mathrm{H} 2 \mathrm{O}}$ was $2 \times 10^{-3} \mathrm{Kg} . \mathrm{Cp}_{, \mathrm{H} 2 \mathrm{O}}$ was $4.2 \times 10^{3} \mathrm{~J} /\left(\mathrm{kg} \cdot{ }^{\circ} \mathrm{C}\right) . \theta$ is the dimensionless driving force temperature,and defined as the ratio of $\Delta T$ to $\Delta T_{\text {max,mix. }}$

\section{Dox loading and release test.}

In order to load the DOX, HA-PDA@IQ NPs ( $1 \mathrm{mg} / \mathrm{mL})$ were dissolved in PBS $(20 \mathrm{mM})$ at pH 7.4. Then, DOX at distinct different weight ratio with HA-PDA@IQ $(0.1,0.2,0.5,1,2,3,3.5)$ were added and stirred for 24 hours in the dark. The above solutions were centrifuged and washed with PBS solution until the supernatants were colorless. The loading capacity of DOX was detected by UV-Vis-NIR spectrum.

Then, the release of DOX from HA-PDA@IQ/DOX was studied under pH 5.0 and 7.4 at different times. The amount of DOX release was also measured by UV-Vis-NIR spectroscopy. In addition, the release of DOX was simulated by NIR under 808 nm laser irradiation. In the experiment, HA-PDA@IQ/DOX was dissolved in $15 \mathrm{~mL}$ PBS with different $\mathrm{pH}$ values (5.0 and 7.4). At different time points, $808 \mathrm{~nm}$ laser was used to irradiate with a power density of $2 \mathrm{~W} / \mathrm{cm}^{2}$ for 5 minutes. Afterwards, $1 \mathrm{~mL}$ of the solution was collected and centrifuged to obtain the released DOX, which was measured and determined by UV-Vis spectroscopy.

\section{Biocompatibility of HA-PDA@IQ NPs}

The CCK-8 assay was used to evaluate the biocompatibility of HA-PDA@IQ NPs by incubating with 4T1 cells (mouse breast cancer cell line) and MEF cells (mouse embryo fibroblast cell line) respectively. Both of cells were inoculated in a 96-well plate with approximately $1 \times 10^{4}$ cells in each well at $37^{\circ} \mathrm{C}$ and $5 \%$ $\mathrm{CO}_{2}$. When the cell density reached $60 \%$, the medium was discarded and replaced with new medium containing different concentrations of PDA@IQ NPs $(0,200,400,600,800,1000,1200 \mu \mathrm{g} / \mathrm{mL})$. After 
incubation for 48 hours, these cells were washed with PBS and incubated with $10 \mu \mathrm{L}$ CCK-8 and $90 \mu \mathrm{L}$ DMEM or RPMI-1640. After 2 hours, the absorbance value of the solution at $450 \mathrm{~nm}$ was measured by ultraviolet spectrophotometer (BioTek Instruments, Inc Vermont, USA). The experiment was repeated with three parallel groups. The relative cell viability was calculated as (Abs sample $-A$ Abs $\left._{\text {zero sitting }}\right) /(A b s$ control $^{-}$Abs zerositting $) \times 100 \%$.

\section{Hemolysis test}

First, $100-150 \mu \mathrm{L}$ of $10 \%$ chloral hydrate solution was administrated into the mouse by intraperitoneal injection. After the deeply anesthetizing, the mouse's eyeballs were removed with tweezers. The fresh eye blood of the mouse was quickly collected into a centrifuge tube with heparin-infiltrated tube wall and centrifuged for 15 minutes at a speed of $1200 \mathrm{rpm}$. The supernatant was discarded and an appropriate amount of PBS was added into the centrifuge tube to wash twice until solution was clear. Finally, the below red precipitate was mouse red blood cells (MRBCs). $100 \mu \mathrm{L}$ of the diluted MRBCs was added into the EP tube, mixed with $900 \mu \mathrm{L}$ of HA-PDA@ IQ/DOX with different concentrations $(100,200,400,800$, $1000,1200 \mu \mathrm{g} / \mathrm{mL}$ ), PBS as negative control, de-ionized water as positive control. All samples were allowed to stand at room temperature for 2 hours, then centrifuged at $1000 \mathrm{rpm}$ for 1 minute. The supernatant was carefully transferred to the new EP tube and measured the absorbance using a dualbeam UV-Vis spectrophotometer at $541 \mathrm{~nm}$.

\section{Phagocytosis experiment}

Confocal laser scanning was performed to study the phagocytosis and distribution of HA-PDA@IQ/DOX in cells. A small disc was pre-installed at the bottom of the 24-well plate. Then, $4 \mathrm{~T} 1$ cells were seeded into the 24-well plate at $1 \times 10^{4}$ cells per well in an incubator at $37^{\circ} \mathrm{C}$ and $5 \% \mathrm{CO}_{2}$ for 24 hours. When the cell density reached $60 \%$, the medium was replaced with new medium containing $50 \mu \mathrm{g} / \mathrm{mL} \mathrm{HA-}$ PDA@IQ/DOX NPs under the same conditions for 6 hours. Next, the medium containing HA-PDA@IQ/DOX nanoparticles was discarded and washed twice with PBS. $0.5 \mathrm{~mL}$ of $4 \%$ paraformaldehyde was added into each well to fix for 15 minutes at room temperature. After washed 3 times with PBS, $300 \mu \mathrm{L}$ DAPI working solution was added to each well and stained for 5 minutes at room temperature in the dark. The cells were washed 3 times with PBS for 10 minutes each. Finally, the small discs were taken out and placed upside down on a glass slide pre-dropped with appropriate glycerin, sealed with a neutral resin and stored at $4{ }^{\circ} \mathrm{C}$ in the dark. These samples were observed under confocal laser scanning microscopy.

\section{DC maturation in vitro}

Bone marrow-derived dendritic cells (BMDCs) were obtained from bone marrow precursors of 6-week-old Balb/c female mice [39]. Then, BMDCs were seed in a 6 -well plate $\left(1 \times 10^{6}\right.$ cells/well). For in vitro stimulation experiments, BMDC was co-cultured with HA-PDA@IQ NPs at different concentrations for 48 hours. DCs were collected and stained with anti-CD11c FITC, anti-CD86 PE and anti-CD80 APC for flow cytometry analysis. 


\section{Mouse tumor model}

In our experiment, female Balb/c mice were purchased from Jiangsu ALF Biotechnology Co., LTD. Animal experiments were carried out in accordance with the protocol approved by the Experimental Animal Center of Jiangsu University. To establish a tumor model, 4T1 cells $\left(1 \times 10^{6}\right)$ in $20 \mu \mathrm{L}$ PBS were subcutaneously injected into the lower left breast of each mouse. After around one week, the average size of the tumor reached about $100 \mathrm{~mm}^{3}$. For in vivo combination therapy, 4T1 tumor-bearing mice were divided into five groups, including PBS, HA-PDA NPs, HA-PDA@IQ NPs, HA-PDA@IQ/DOX NPs and HAPDA@IQ/DOX HG. The tumor was irradiated with $808 \mathrm{~nm}$ laser at a power density of $2 \mathrm{~W} / \mathrm{cm}^{2}$ for 5 minutes. During the laser irradiation, the temperature change of the tumor was recorded by an infrared thermal imaging camera (HT-19). After treatment, Tumor size was monitored by vernier caliper to record the lengths and widths every two days for two weeks. The tumor volumes were calculated by "length $x$ width ${ }^{2} / 2^{\prime \prime}$.

\section{MR imaging}

The Skyra 3.0T superconducting magnetic resonance imaging system (MAGNETOM Trio TimSystem, Siemens, Germany) was used to scan different concentrations of HA-PDA@IQ/DOX NPs aqueous solution (0-0.20 nM Mn). $T_{1}$-weighted animal MR imaging was performed on the same MR scanner with a special coil specially designed for small animal images, the images were obtained under the following parameters: $\mathrm{TR} / \mathrm{TE}=600 / 9.2 \mathrm{~ms}$, slices thickness $=2 \mathrm{~mm}, 256 \times 256$ matrices .

\section{Histological analysis}

In order to conduct biocompatibility studies, five groups of mice with different treatments were sacrificed and the major organs (heart, spleen, liver, lung, kidney, and tumor) were collected. These organs were immersed in $4 \%$ paraformaldehyde buffer solution for 16 hours, then trimmed, dehydrated, transparent treated and embedded in paraffin wax. The samples were sliced serially at a thickness of $5 \mu \mathrm{m}$ and stained with hematoxylin and eosin. Finally, observe the tissue section under an optical microscope.

\section{Statistical analysis}

All experiments were repeated at least three times, and the results were expressed as mean \pm SD. The statistical significance of all results was determined by the student's $t$ test. $P<0.05$ was considered statistically significant.

\section{Declarations}

\section{Ethics approval and consent to participate}

All experimental procedures were approved by the Institutional Animal Care and Use Committee of Medical College of Jiangsu University (NO. UJS-IACUC-AP-20190314002). 


\section{Consent for publication}

Each coauthor has read the manuscript and approves its submission, and this work is being submitted exclusively to your journal.

\section{Availability of data and material}

The data sets supporting the results of this article are included within the article.

\section{Competing interests}

All authors claim no conflict of interest.

\section{Funding}

The research was supported by Natural Science Foundation of Jiangsu Province (SBK2020022937, BK20161317), Key talents of young medicine in Jiangsu Province (QNRC2016444), Six talent peaks project in Jiangsu Province (No: WSN-281), Zhenjiang key research and development plan (SH2019002), Major natural science research projects of colleges and universities in Jiangsu province (19KJA150004).

\section{Authors' contributions}

Lirong Zhang and Jinajing Zhang are responsive for experimental operation. Lixia Xu, Zijian Zhuang, Jingjin Liu, Suwan Liu are responsive for data collection and analysis. Yunchao Wu, Aihua Gong are responsive for data processing and drawing. Fengyi Du and Miaomiao Zhang are responsive for experimental design and writing.

\section{Acknowledgements}

The Acknowledged are included within the article.

\section{References}

1. Gong N., Zhang Y., Teng X., Wang Y., Huo S., Qing G., Ni Q., Li X., Wang J., Ye X., Zhang T., Chen S., Wang Y., Yu J., Wang P. C., Gan Y., Zhang J., Mitchell M. J., Li J., Liang X. J. Proton-driven transformable nanovaccine for cancer immunotherapy. Nat Nanotechnol [J]. 2020; 15: 1053-1064.

2. Shin H., Na K. In situ vaccination with biocompatibility controllable immuno-sensitizer inducing antitumor immunity. Biomaterials [J]. 2019; 197: 32-40.

3. Xie X., Hu Y., Ye T., Chen Y., Zhou L., Li F., Xi X., Wang S., He Y., Gao X., Wei W., Ma G., Li Y. Therapeutic vaccination against leukaemia via the sustained release of co-encapsulated anti-PD-1 and a leukaemia-associated antigen. Nat Biomed Eng [J]. 2020.

4. Hu Z., Ott P. A., Wu C. J. Towards personalized, tumour-specific, therapeutic vaccines for cancer. Nat Rev Immunol [J]. 2018; 18: 168-182. 
5. Tau N., Yahav D., Shepshelovich D. Postmarketing Safety of Vaccines Approved by the U.S. Food and Drug Administration : A Cohort Study. Ann Intern Med [J]. 2020; 173: 445-449.

6. Saif J. M., Vadakekolathu J., Rane S. S., Mcdonald D., Ahmad M., Mathieu M., Pockley A. G., Durrant L., Metheringham R., Rees R. C., Mcardle S. E. Novel prostate acid phosphatase-based peptide vaccination strategy induces antigen-specific T-cell responses and limits tumour growth in mice. Eur J Immunol [J]. 2014; 44: 994-1004.

7. Zou M., Jin R., Au K. F. Revealing tumor heterogeneity of breast cancer by utilizing the linkage between somatic and germline mutations. Brief Bioinform [J]. 2019; 20: 2306-2315.

8. Parkhurst M. R., Robbins P. F., Tran E., Prickett T. D., Gartner J. J., Jia L., Ivey G., Li Y. F., El-Gamil M., Lalani A., Crystal J. S., Sachs A., Groh E., Ray S., Ngo L. T., Kivitz S., Pasetto A., Yossef R., Lowery F. J., Goff S. L., Lo W., Cafri G., Deniger D. C., Malekzadeh P., Ahmadzadeh M., Wunderlich J. R., Somerville R. P. T., Rosenberg S. A. Unique Neoantigens Arise from Somatic Mutations in Patients with Gastrointestinal Cancers. Cancer Discov [J]. 2019; 9: 1022-1035.

9. Decordova S., Shastri A., Tsolaki A. G., Yasmin H., Klein L., Singh S. K., Kishore U. Molecular Heterogeneity and Immunosuppressive Microenvironment in Glioblastoma. Front Immunol [J]. 2020; 11: 1402.

10. Ni K., Lan G., Guo N., Culbert A., Luo T., Wu T., Weichselbaum R. R., Lin W. Nanoscale metal-organic frameworks for X-ray activated in situ cancer vaccination. Sci Adv [J]. 2020; 6: eabb5233.

11. Patel R. B., Ye M., Carlson P. M., Jaquish A., Zangl L., Ma B., Wang Y., Arthur I., Xie R., Brown R. J., Wang X., Sriramaneni R., Kim K., Gong S., Morris Z. S. Development of an In Situ Cancer Vaccine via Combinational Radiation and Bacterial-Membrane-Coated Nanoparticles. Adv Mater [J]. 2019; 31: e1902626.

12. Liu Q., Cai J., Zheng Y., Tan Y., Wang Y., Zhang Z., Zheng C., Zhao Y., Liu C., An Y., Jiang C., Shi L., Kang C., Liu Y. NanoRNP Overcomes Tumor Heterogeneity in Cancer Treatment. Nano Lett [J]. 2019; 19: 7662-7672.

13. Gilliet M., Cao W., Liu Y. J. Plasmacytoid dendritic cells: sensing nucleic acids in viral infection and autoimmune diseases. Nat Rev Immunol [J]. 2008; 8: 594-606.

14. Park A. K., Fong Y., Kim S. I., Yang J., Murad J. P., Lu J., Jeang B., Chang W. C., Chen N. G., Thomas S. H., Forman S. J., Priceman S. J. Effective combination immunotherapy using oncolytic viruses to deliver CAR targets to solid tumors. Sci Transl Med [J]. 2020; 12: eaaz1863.

15. Weichselbaum R. R., Liang H., Deng L., Fu Y. X. Radiotherapy and immunotherapy: a beneficial liaison? Nat Rev Clin Oncol [J]. 2017; 14: 365-379.

16. Gao S., Li T., Guo Y., Sun C., Xianyu B., Xu H. Selenium-Containing Nanoparticles Combine the NK Cells Mediated Immunotherapy with Radiotherapy and Chemotherapy. Adv Mater [J]. 2020; 32: e1907568.

17. Mai X., Zhang Y., Fan H., Song W., Chang Y., Chen B., Shi J., Xin X., Teng Z., Sun J., Teng G. Integration of immunogenic activation and immunosuppressive reversion using mitochondrial-respirationinhibited platelet-mimicking nanoparticles. Biomaterials [J]. 2020; 232: 119699. 
18. Wu C., Guan X., Xu J., Zhang Y., Liu Q., Tian Y., Li S., Qin X., Yang H., Liu Y. Highly efficient cascading synergy of cancer photo-immunotherapy enabled by engineered graphene quantum dots/photosensitizer/CpG oligonucleotides hybrid nanotheranostics. Biomaterials [J]. 2019; 205: 106-119.

19. Xie Z., Fan T., An J., Choi W., Duo Y., Ge Y., Zhang B., Nie G., Xie N., Zheng T., Chen Y., Zhang H., Kim J. $\mathrm{S}$. Emerging combination strategies with phototherapy in cancer nanomedicine. Chem Soc Rev [J]. 2020; 49: 8065-8087.

20. Walters A. A., Wang J. T., Al-Jamal K. T. Evaluation of cell surface reactive immuno-adjuvant in combination with immunogenic cell death inducing drug for in situ chemo-immunotherapy. J Control Release [J]. 2020; 322: 519-529.

21. Jaime-Sanchez P., Uranga-Murillo I., Aguilo N., Khouili S. C., Arias M. A., Sancho D., Pardo J. Cell death induced by cytotoxic CD8(+) T cells is immunogenic and primes caspase-3-dependent spread immunity against endogenous tumor antigens. J Immunother Cancer [J]. 2020; 8: e000528.

22. Zhou L., Zhang P., Wang H., Wang D., Li Y. Smart Nanosized Drug Delivery Systems Inducing Immunogenic Cell Death for Combination with Cancer Immunotherapy. Acc Chem Res [J]. 2020; 53: 1761-1772.

23. Qin Jing, Liang Guohai, Feng Youyou, Feng Bingxi, Wang Gen, Wu Na, Zhao Yongxi, Wei Jing. Synthesis of gadolinium/iron-bimetal-phenolic coordination polymer nanoparticles for theranostic applications. Nanoscale [J]. 2020; 12: 6096-6103.

24. Tian Dan, Xu Hongxia, Xiao Bing, Zhou Xiaoxuan, Liu Xiangrui, Zhou Zhuxian, Patra Hirak K., Slater Nigel, Tang Jianbin, Shen Youqing. Single-step formulation of levodopa-based nanotheranostics strategy for ultra-sensitive high longitudinal relaxivity MRI guided switchable therapeutics. Biomaterials Science [J]. 2020; 8: 1615-1621.

25. Zhou J., Zhao W., Miao Z., Wang J., Ma Y., Wu H., Sun T., Qian H., Zha Z. Folin-Ciocalteu Assay Inspired Polyoxometalate Nanoclusters as a Renal Clearable Agent for Non-Inflammatory Photothermal Cancer Therapy. ACS Nano [J]. 2020; 14: 2126-2136.

26. Li M., Sun W., Tian R., Cao J., Tian Y., Gurram B., Fan J., Peng X. Smart J-aggregate of cyanine photosensitizer with the ability to target tumor and enhance photodynamic therapy efficacy. Biomaterials [J]. 2020; 4: 120532.

27. Wang F., Su H., Xu D., Dai W., Zhang W., Wang Z., Anderson C. F., Zheng M., Oh R., Wan F., Cui H. Tumour sensitization via the extended intratumoural release of a STING agonist and camptothecin from a self-assembled hydrogel. Nat Biomed Eng [J]. 2020; 4: 1090-1101.

28. Song C., Phuengkham H., Kim Y. S., Dinh V. V., Lee I., Shin I. W., Shin H. S., Jin S. M., Um S. H., Lee H., Hong K. S., Jin S. M., Lee E., Kang T. H., Park Y. M., Lim Y. T. Syringeable immunotherapeutic nanogel reshapes tumor microenvironment and prevents tumor metastasis and recurrence. Nat Commun [J]. 2019; 10: 3745.

29. Sagiv-Barfi I., Czerwinski D. K., Levy S., Alam I. S., Mayer A. T., Gambhir S. S., Levy R. Eradication of spontaneous malignancy by local immunotherapy. Sci Transl Med [J]. 2018; 10: eaan4488. 
30. Klinman D. M. Immunotherapeutic uses of $\mathrm{CpG}$ oligodeoxynucleotides. Nat Rev Immunol [J]. 2004; 4: 249-258.

31. Alexander-Miller M. A., Leggatt G. R., Sarin A., Berzofsky J. A. Role of antigen, CD8, and cytotoxic T lymphocyte (CTL) avidity in high dose antigen induction of apoptosis of effector CTL. J Exp Med [J]. 1996; 184: 485-492.

32. Zhou S., Huang Y., Chen Y., Liu S., Xu M., Jiang T., Song Q., Jiang G., Gu X., Gao X., Chen J. Engineering ApoE3-incorporated biomimetic nanoparticle for efficient vaccine delivery to dendritic cells via macropinocytosis to enhance cancer immunotherapy. Biomaterials [J]. 2020; 235: 119795.

33. Chen Q., Chen J., Yang Z., Xu J., Xu L., Liang C., Han X., Liu Z. Nanoparticle-Enhanced Radiotherapy to Trigger Robust Cancer Immunotherapy. Adv Mater [J]. 2019; 31: e1802228.

34. Keam S., Gill S., Ebert M. A., Nowak A. K., Cook A. M. Enhancing the efficacy of immunotherapy using radiotherapy. Clin Transl Immunology [J]. 2020; 9: e1169.

35. Le Q. V., Suh J., Choi J. J., Park G. T., Lee J. W., Shim G., Oh Y. K. In Situ Nanoadjuvant-Assembled Tumor Vaccine for Preventing Long-Term Recurrence. ACS Nano [J]. 2019; 13: 7442-7462.

36. Gan Shaoju, Tong Xiaoning, Zhang Yue, Wu Jinhui, Hu Yiqiao, Yuan Ahu. Covalent Organic Framework-Supported Molecularly Dispersed Near-Infrared Dyes Boost Immunogenic Phototherapy against Tumors. Advanced Functional Materials [J]. 2019; 29.

37. Li Yuce, Xie Jun, Um Wooram, You Dong Gil, Kwon Seunglee, Zhang Lianbin, Zhu Jintao, Park Jae Hyung. Sono/Photodynamic Nanomedicine-Elicited Cancer Immunotherapy. Advanced Functional Materials [J]. 2020.

38. Wei X., Liu L., Li X., Wang Y., Guo X., Zhao J., Zhou S. Selectively targeting tumor-associated macrophages and tumor cells with polymeric micelles for enhanced cancer chemo-immunotherapy. J Control Release [J]. 2019; 313: 42-53.

39. Liu Y., Ai K., Liu J., Deng M., He Y., Lu L. Dopamine-melanin colloidal nanospheres: an efficient nearinfrared photothermal therapeutic agent for in vivo cancer therapy. Adv Mater [J]. 2013; 25: 13531359.

40. You Y., Cao F., Zhao Y., Deng Q., Sang Y., Li Y., Dong K., Ren J., Qu X. Near-Infrared Light DualPromoted Heterogeneous Copper Nanocatalyst for Highly Efficient Bioorthogonal Chemistry in Vivo. ACS Nano [J]. 2020; 14: 4178-4187.

41. Ren W., Yan Y., Zeng L., Shi Z., Gong A., Schaaf P., Wang D., Zhao J., Zou B., Yu H., Chen G., Brown E. M., Wu A. A Near Infrared Light Triggered Hydrogenated Black TiO2 for Cancer Photothermal Therapy. Adv Healthc Mater [J]. 2015; 4: 1526-1536.

\section{Figures}


a

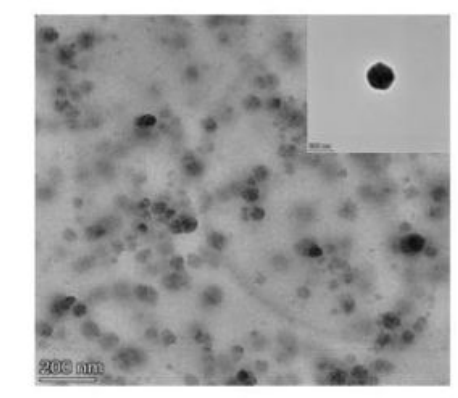

d
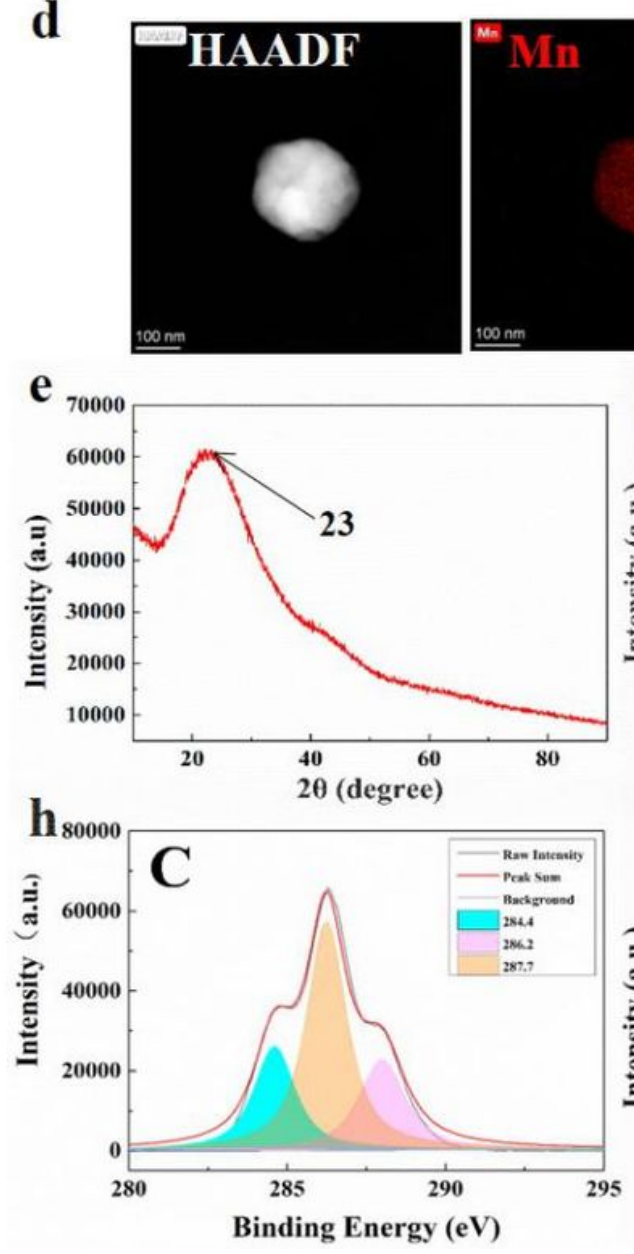

b
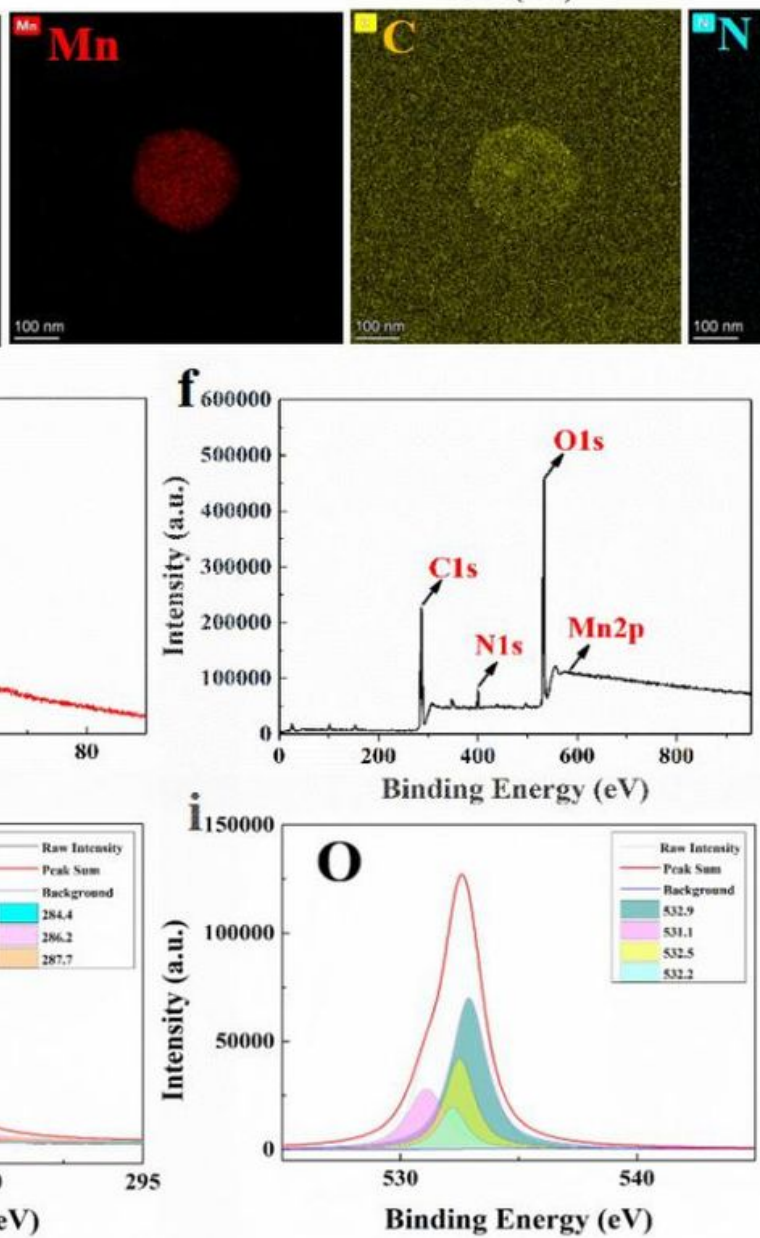

C
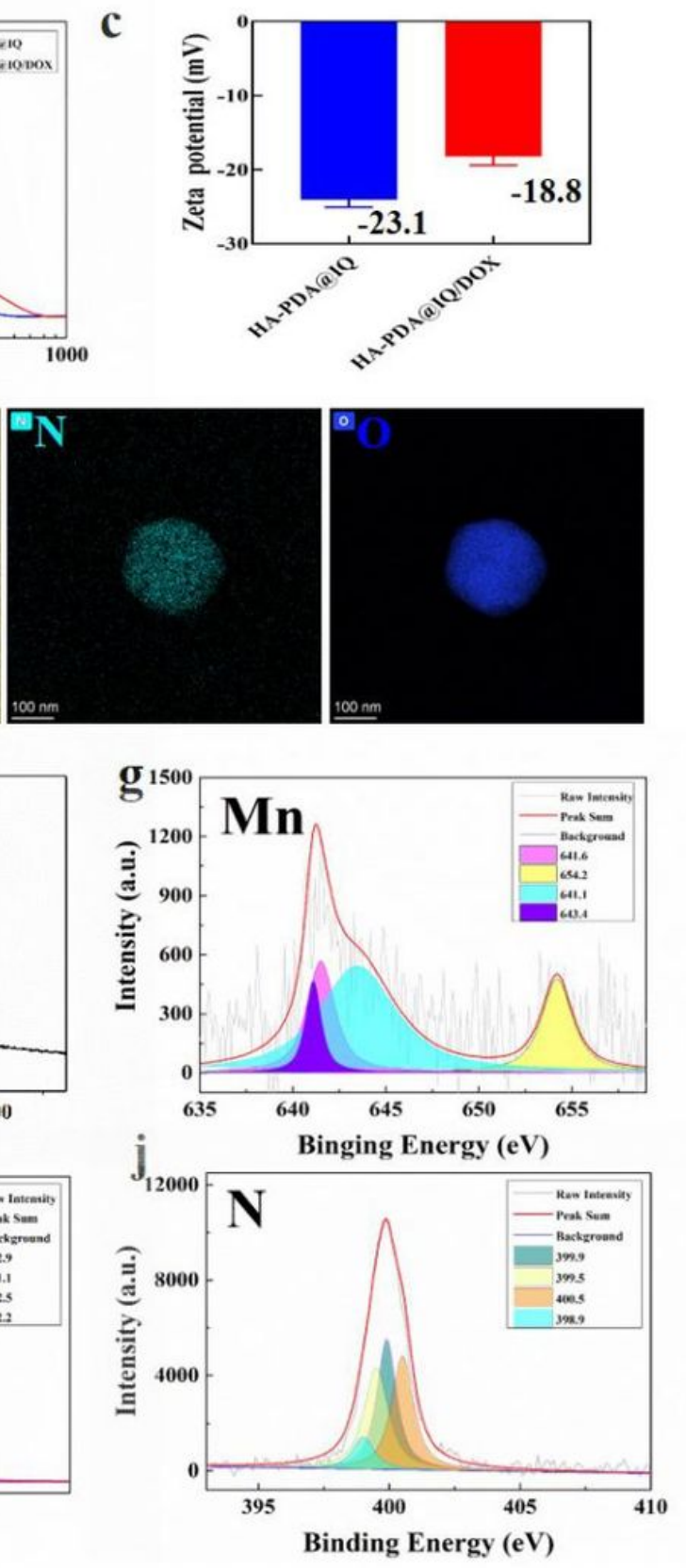

\section{Figure 1}

Characterizations of HA-PDA@IQ/DOX NPs. (a) Typical TEM images, (b) Particle size distribution, (c) Surface zeta potential, (d) FETEM images and elemental mapping images (Mn, C, N, and O), (e) XRD spectra, (f) XPS spectra of HA-PDA@IQ/DOX NPs. (g) Peak-fitting spectra for Mn2p, (h) C1s, (i) 01s, (j) N1s of HA-PDA@IQ/DOX NPs. 
a

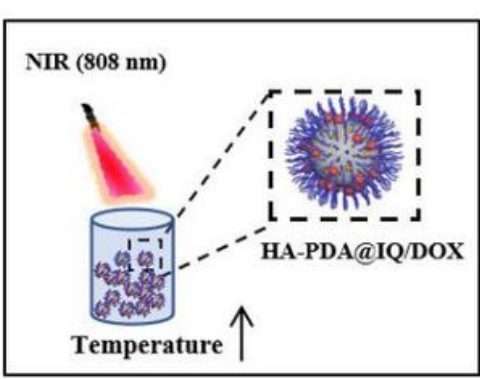

d
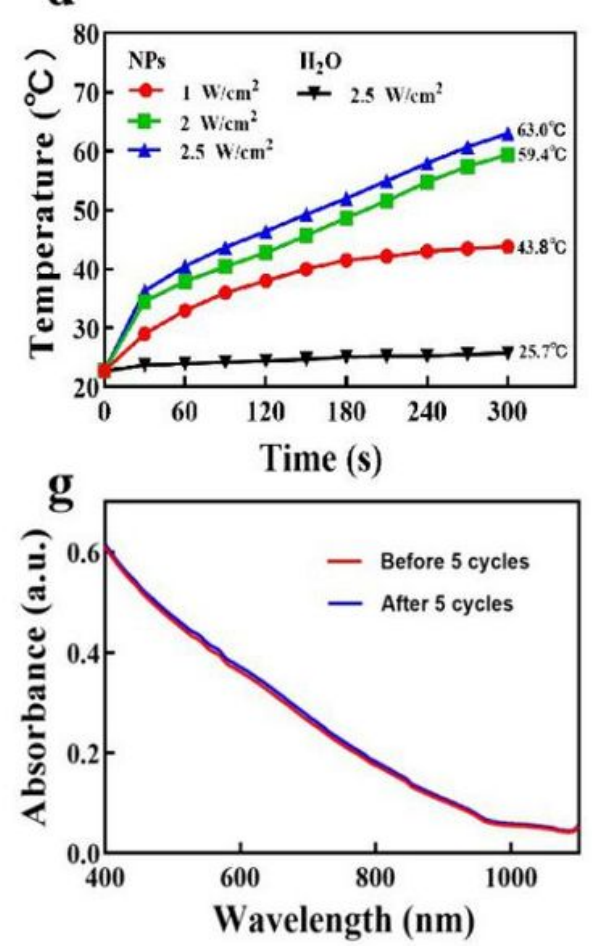
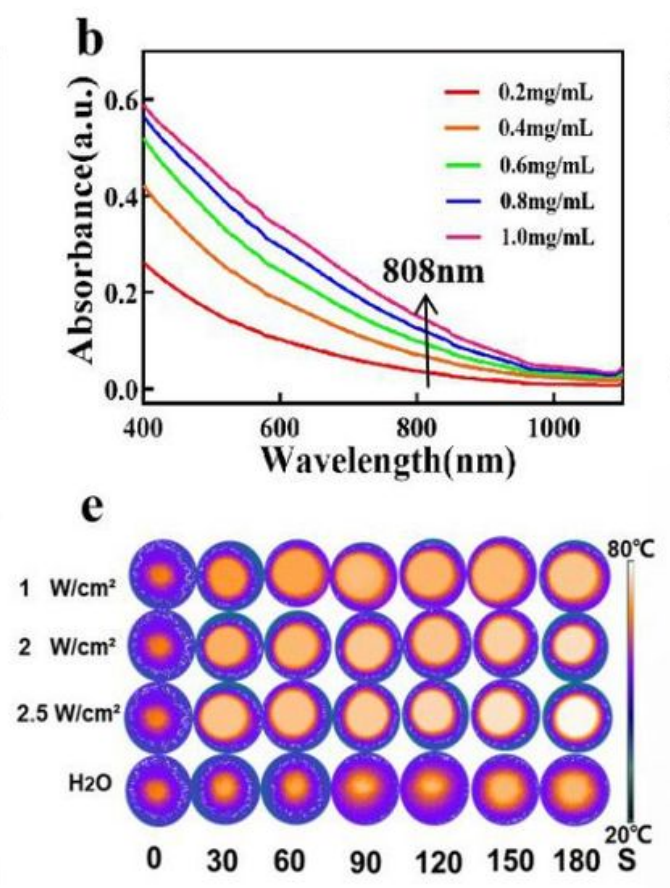

f

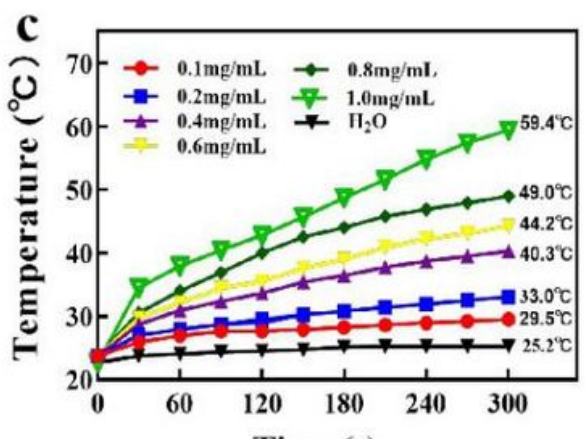

Time (s)

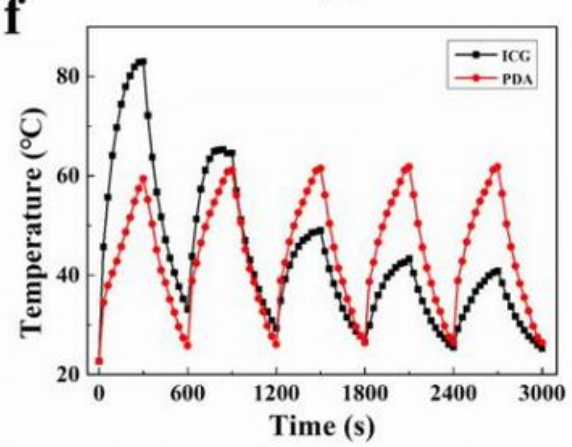

h

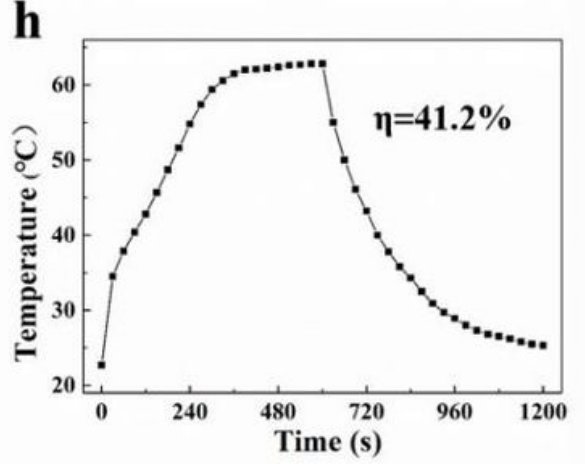

i

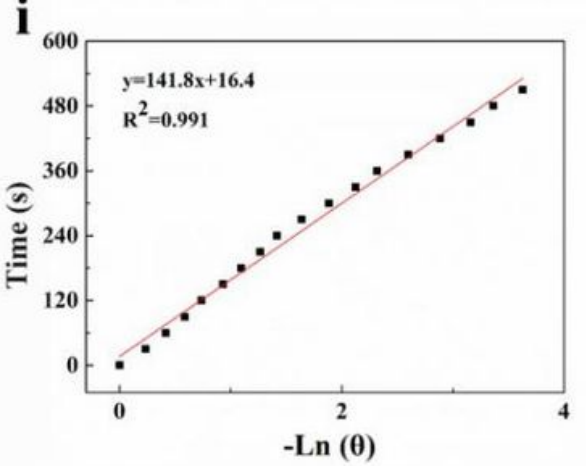

Figure 2

In vitro photothermal performance of HA-PDA@IQ/DOX NPs. (a) Diagram of the photothermal effect of HA-PDA@IQ/DOX NPs. (b) Vis-NIR absorbance spectra of HA-PDA@IQ/DOX. (c) Photothermal heating curves of HA-PDA@IQ/DOX at different concentrations and (d) under varied power densities. (e) Thermal imaging of HA-PDA@IQ/DOX NPs solution under different laser power density. (f) Periodical temperature changes of the HA-PDA@IQ/DOX NPs solution upon laser irradiation for five laser on/off cycles and (g) following Vis-NIR absorbance spectra. (h) Photothermal response of HA-PDA@IQ/DOX NPs solution treated with an NIR laser (808 nm, 2 W/cm2) for $600 \mathrm{~s}$ and left to cool down. (i) Linear time data versus $-\ln \theta$ obtained from the cooling stage. 
a
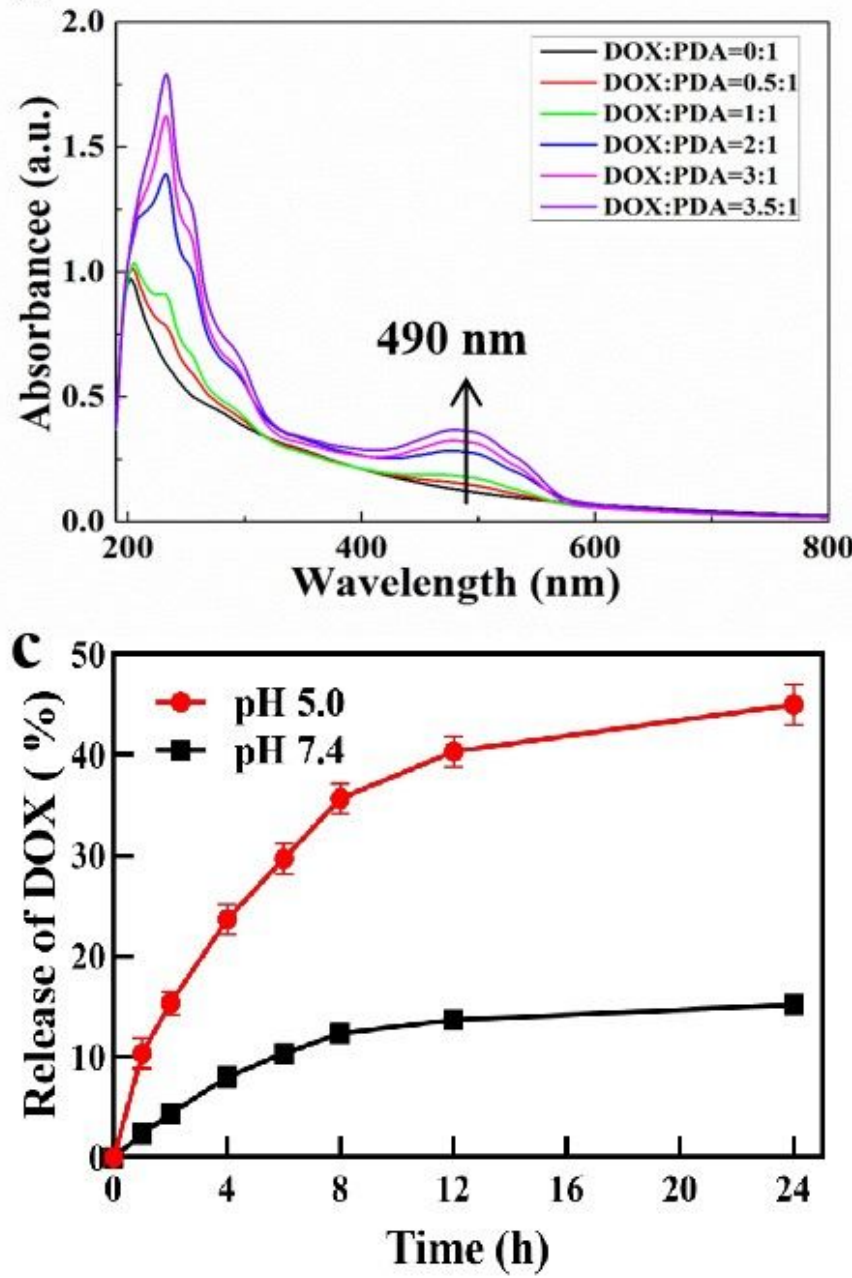

b
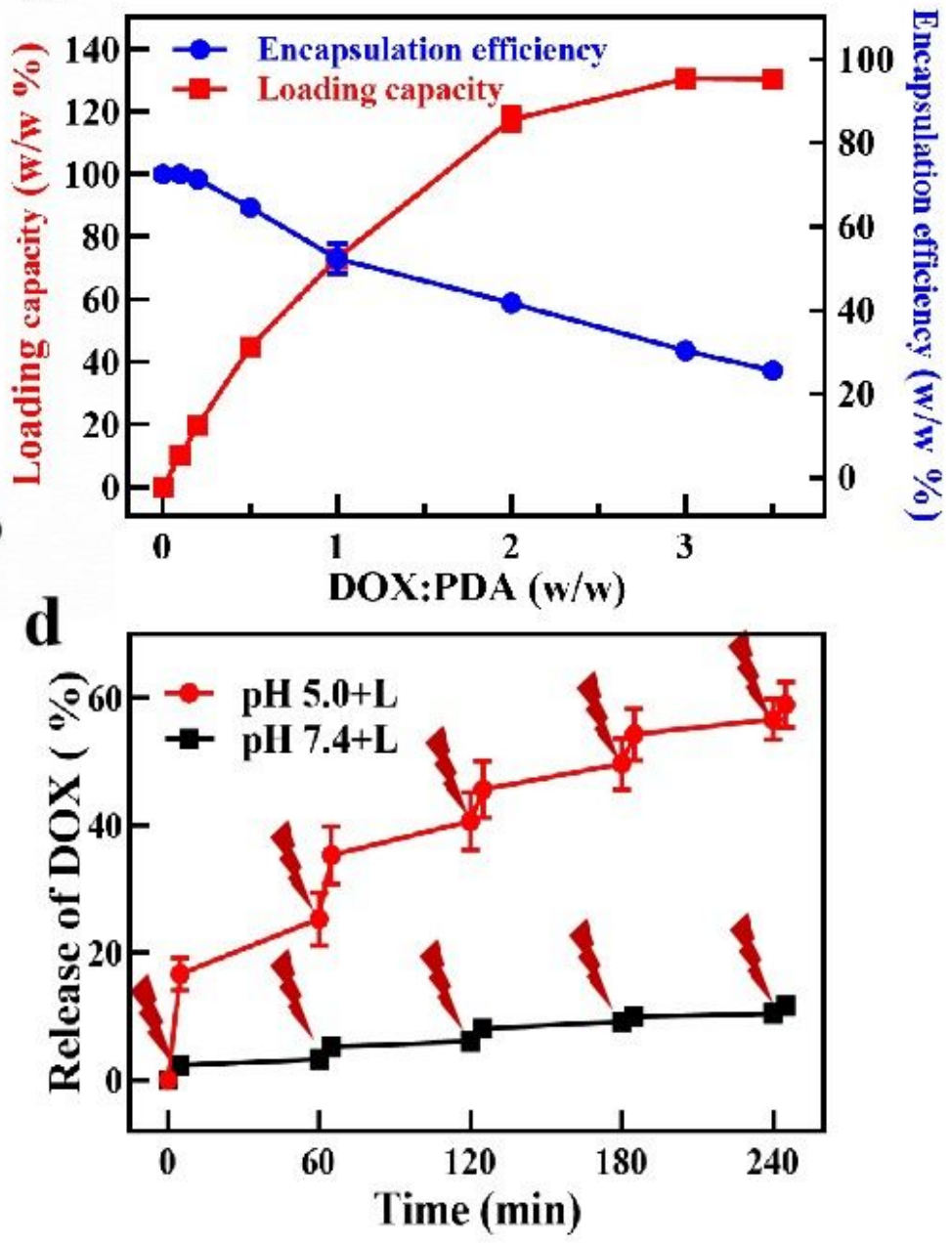

Figure 3

The loading and release profiles of DOX. (a) UV-Vis-NIR spectra of HA-PDA@IQ/DOX NPs with different feeding ratios of DOX to HA-PDA@IQ. (b) Quantification of DOX loading from HA-PDA@IQ/DOX NPs at different ratios of DOX: HA-PDA@IQ. (c) DOX release from HA-PDA@IQ/DOX NPs at the different pH values. (d) NIR-triggered release of DOX from HA-PDA@IQ/DOX NPs. 

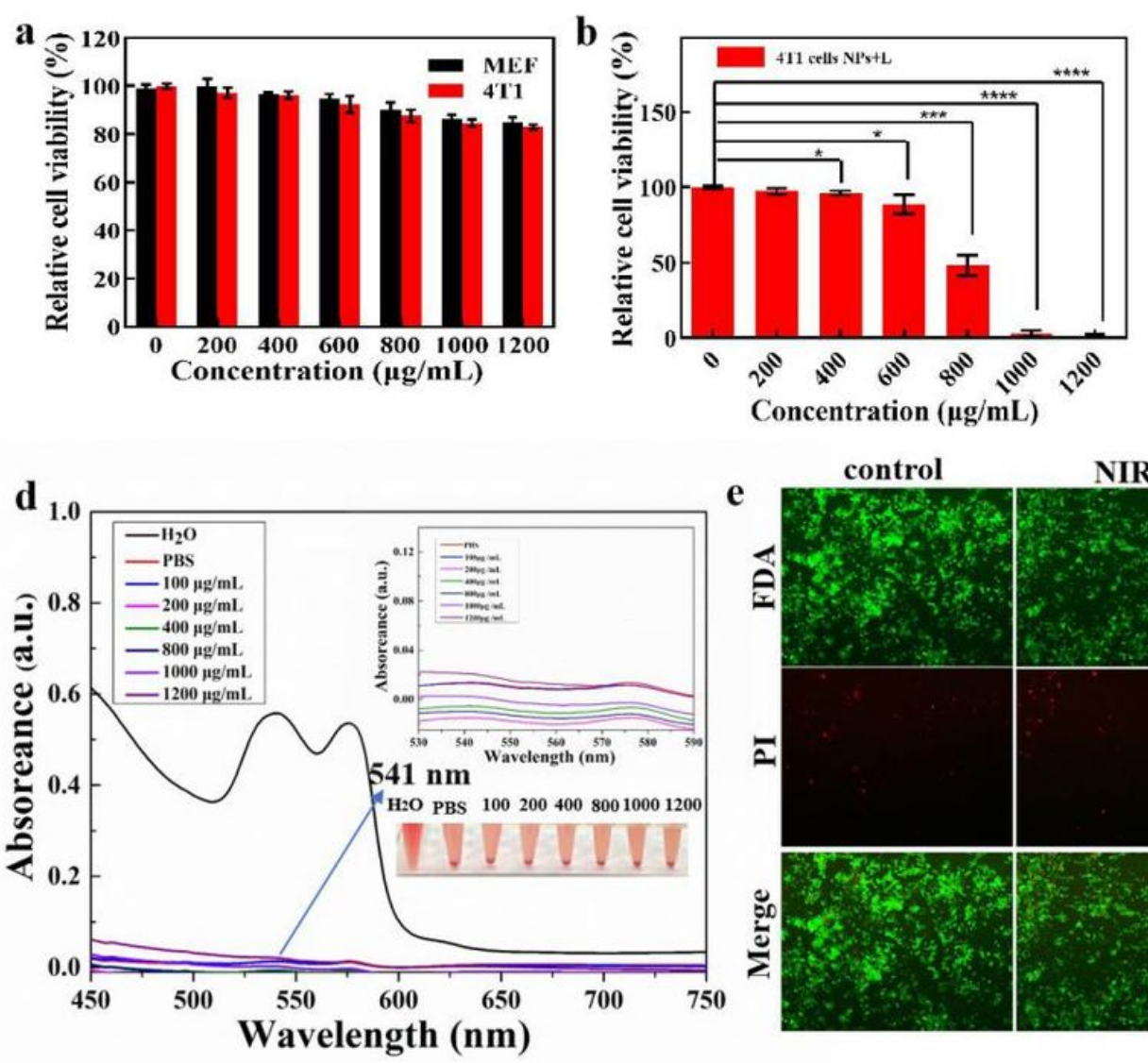
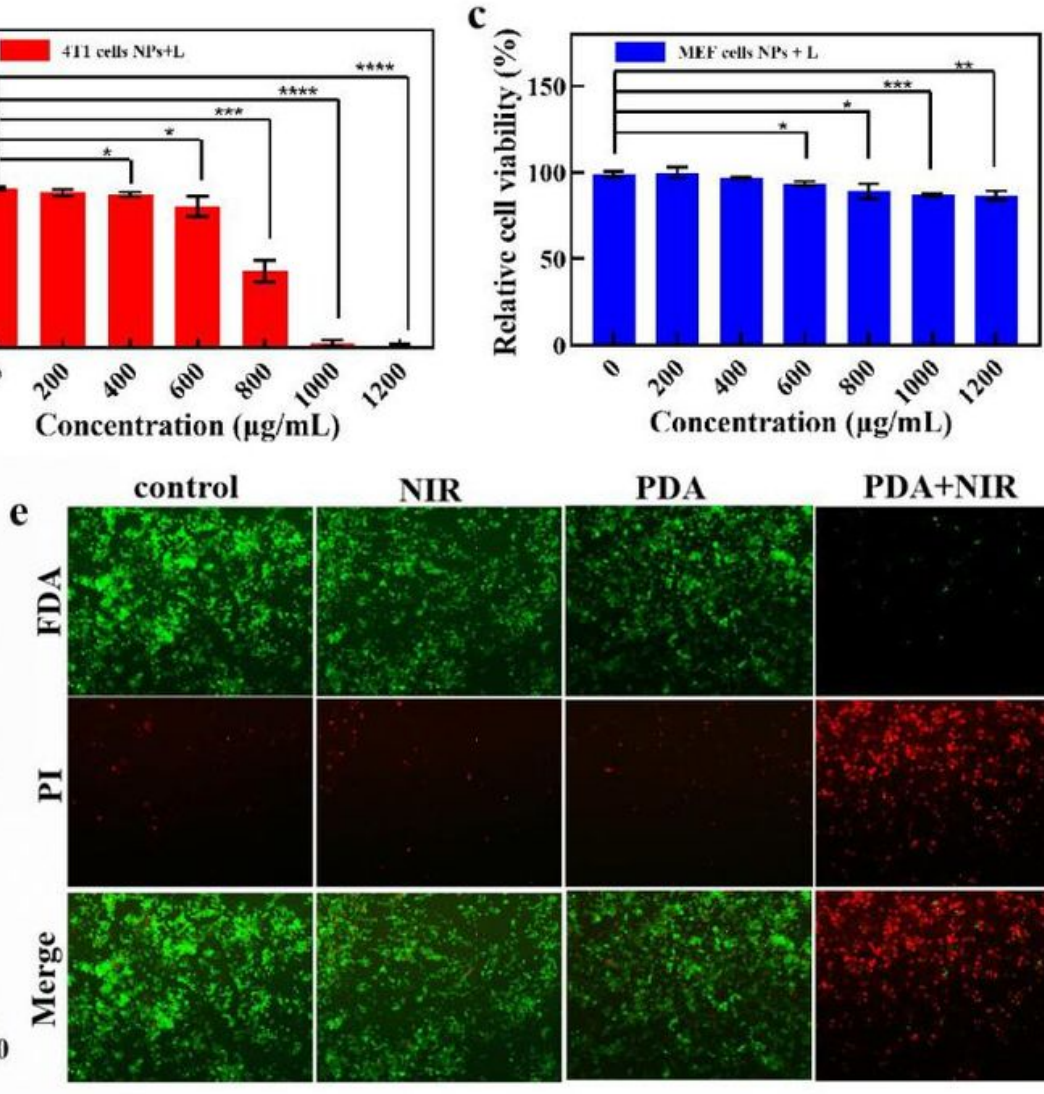

NIR

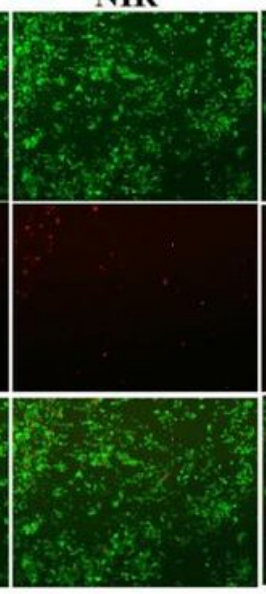

PDA

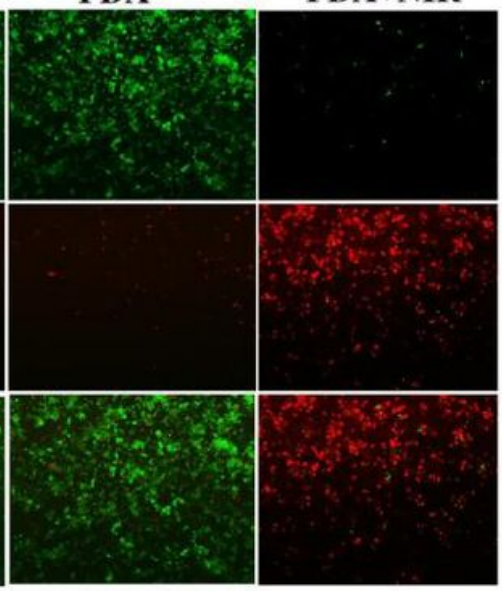

\section{Figure 4}

(a) Cell viability of 4T1 cells and MEF cells co-cultured with different concentrations of HA-PDA@IQ NPs for 48 hours. (b) Relative viability of 4T1 cells and (c) MEF cells after HA-PDA@IQ NPs upon laser irradiation for $48 \mathrm{~h}$ at various concentrations. (d) Hemolytical activity of the HA-PDA@IQ at different concentrations. PBS and water were used as negative and positive control, respectively(e) Fluorescent images of $4 \mathrm{~T} 1$ cells in different treatment groups by live (green) and dead (red) cell staining. 

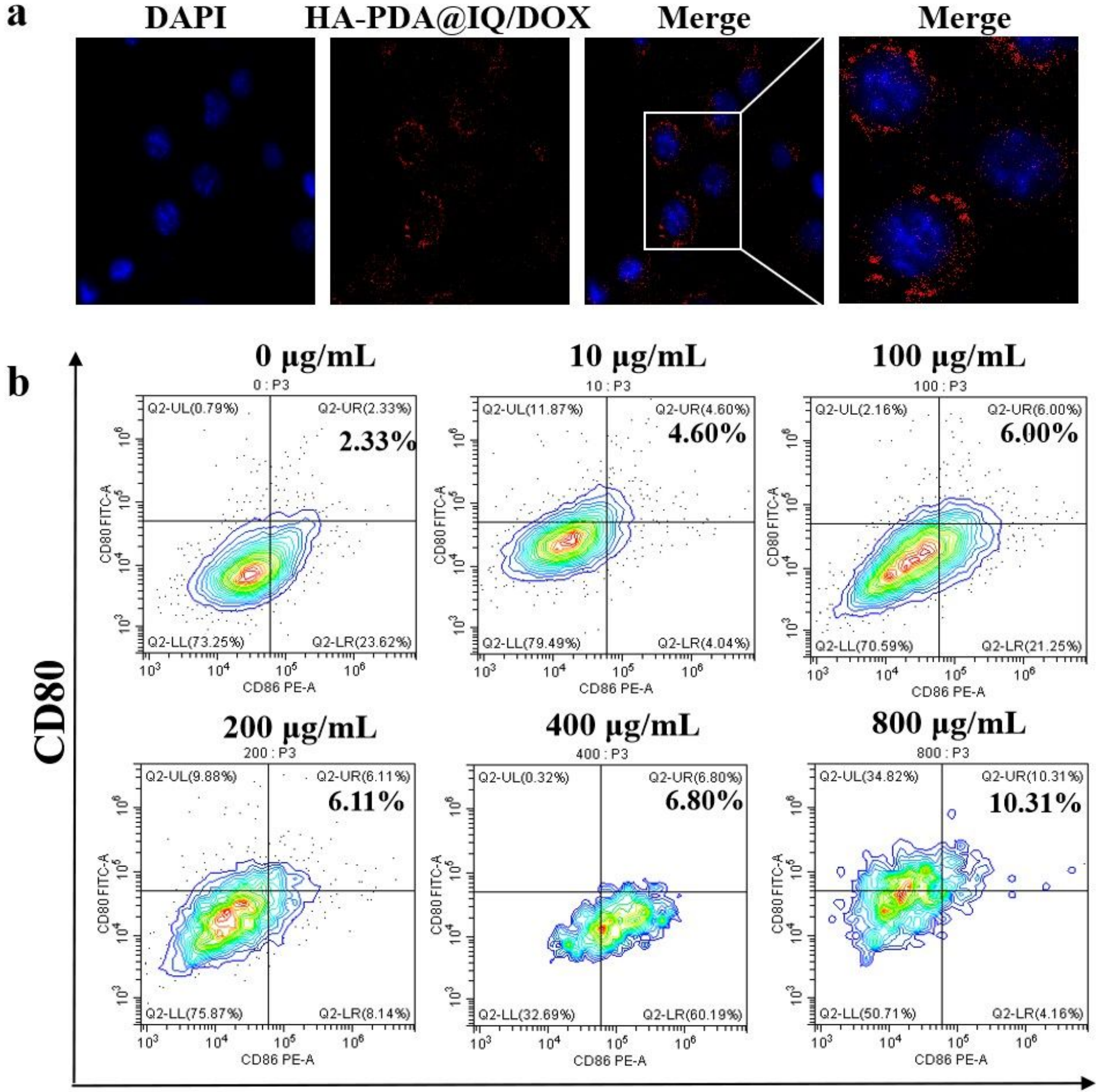

CD86

Figure 5

(a) LSCM images of 4T1cells incubated with $100 \mu \mathrm{g} / \mathrm{ml} \mathrm{HA-PDA@IQ/DOX} \mathrm{NPs.} \mathrm{(b)} \mathrm{Flow} \mathrm{cytometry}$ analysis of CD80 and CD86 on the BMDCs surface after cocultured with HA-PDA@IQ NPs at different concentrations. 

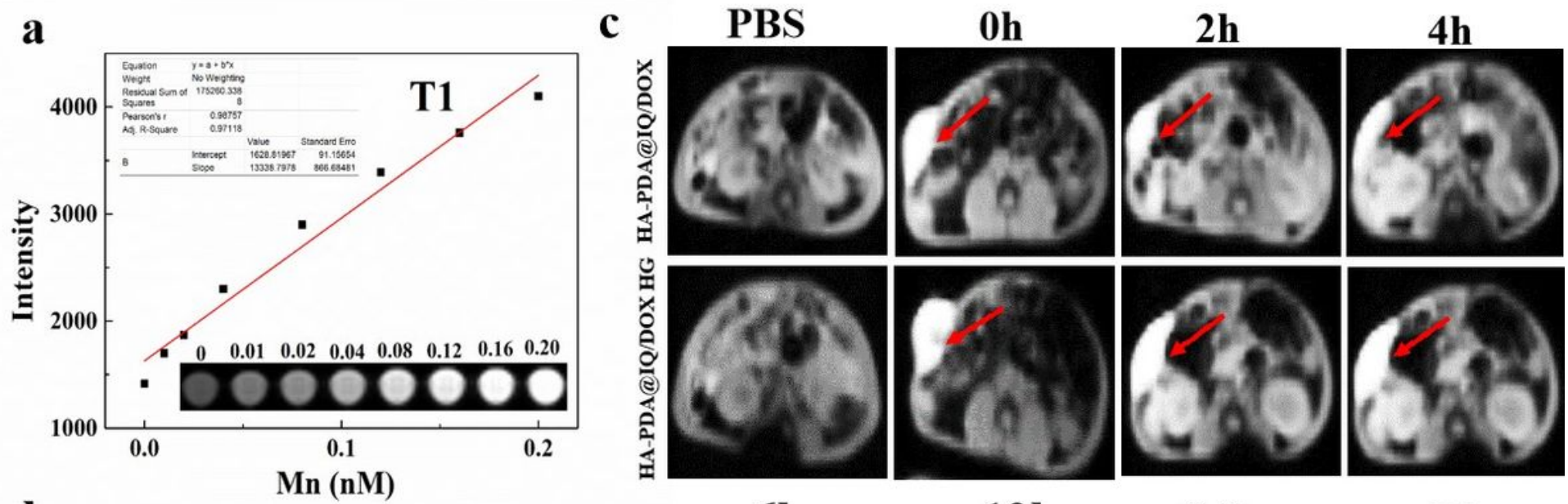

b

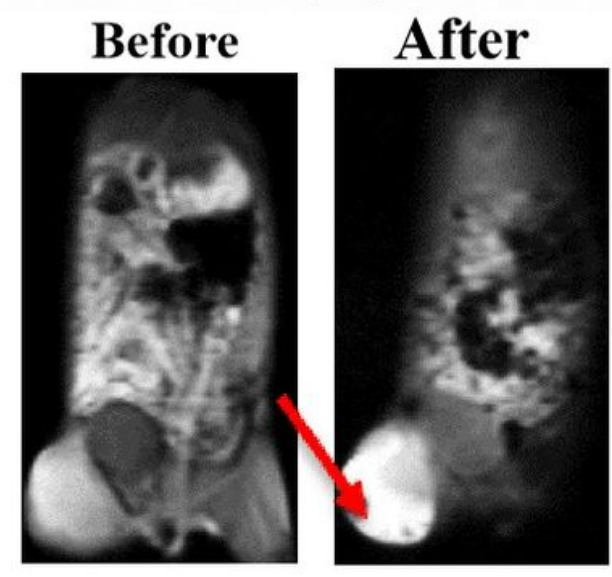

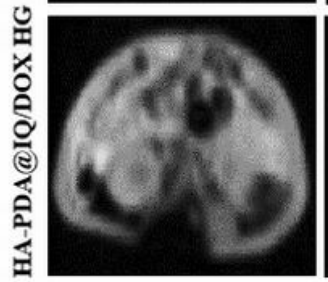
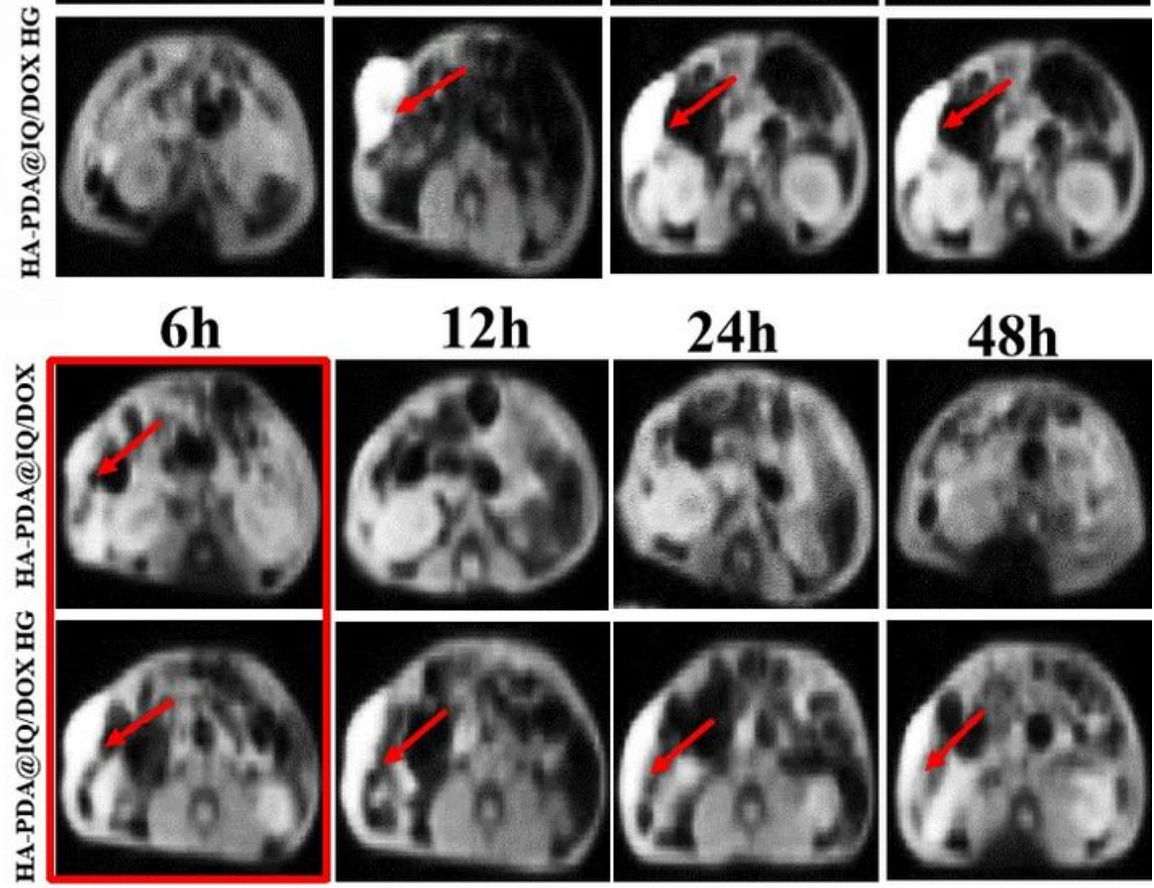
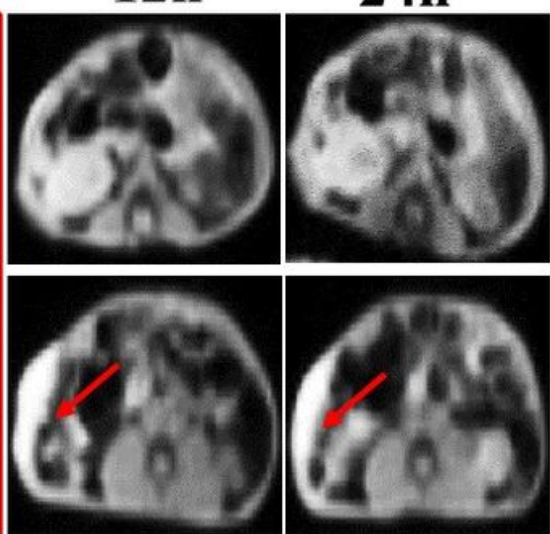

$24 h$

$48 \mathrm{~h}$

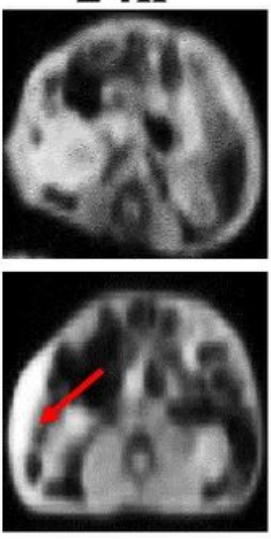

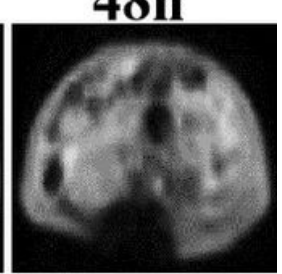

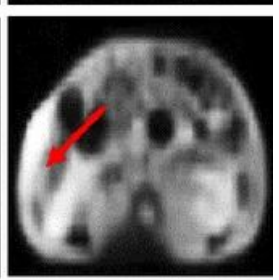

Figure 6

(a) T1-weighted MR images of HA-PDA@IQ/DOX containing various Mn concentration. (b) T1-weighted MR images of HA-PDA@IQ/DOX NPs at the tumor site. (c) T1-weighted images of HA-PDA@IQ/DOX NG retention in the tumor site within 48 hours. 
$\mathbf{a}$

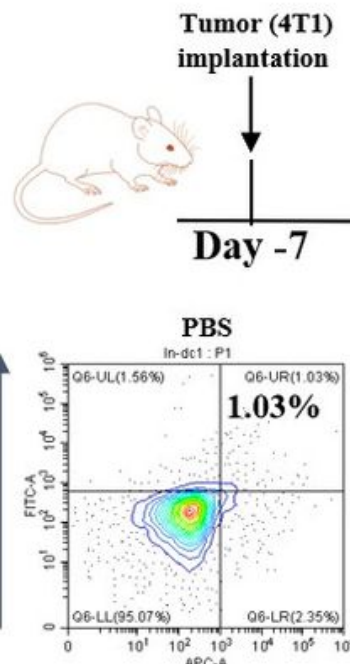

implantation
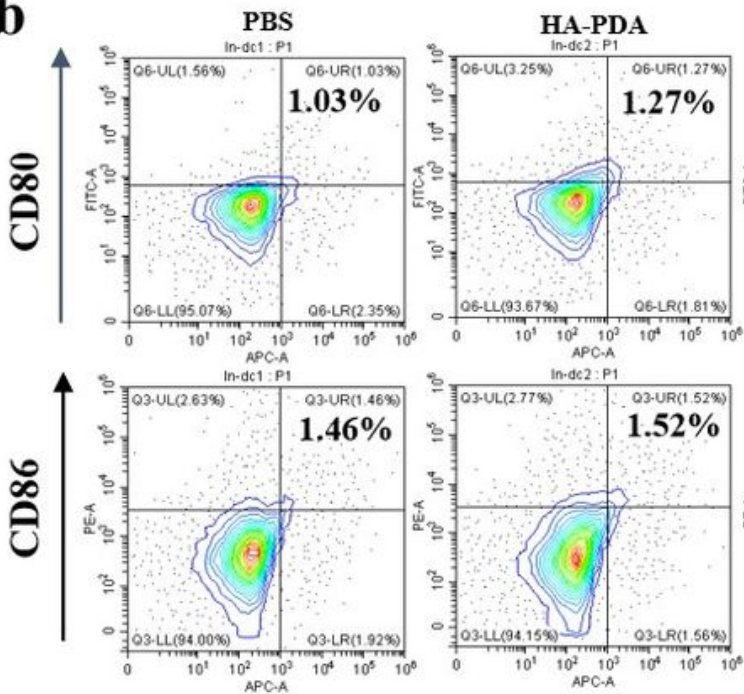

Day 0
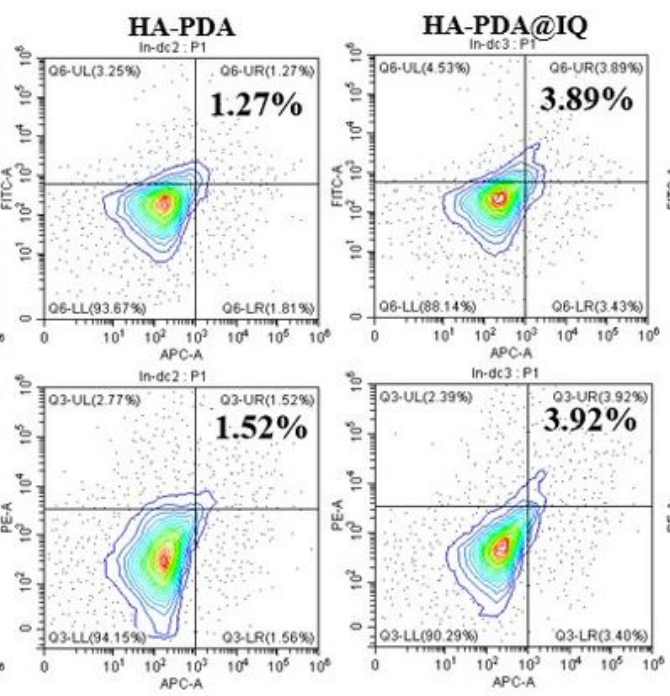

Day 1

+Laser irradiation
In-de1: $P$ I

b

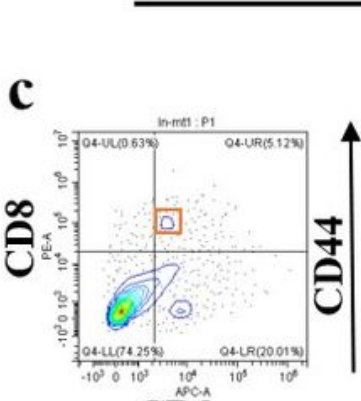

CD3

\section{LN (DCs)}

LN, Spleen excision

Day 7
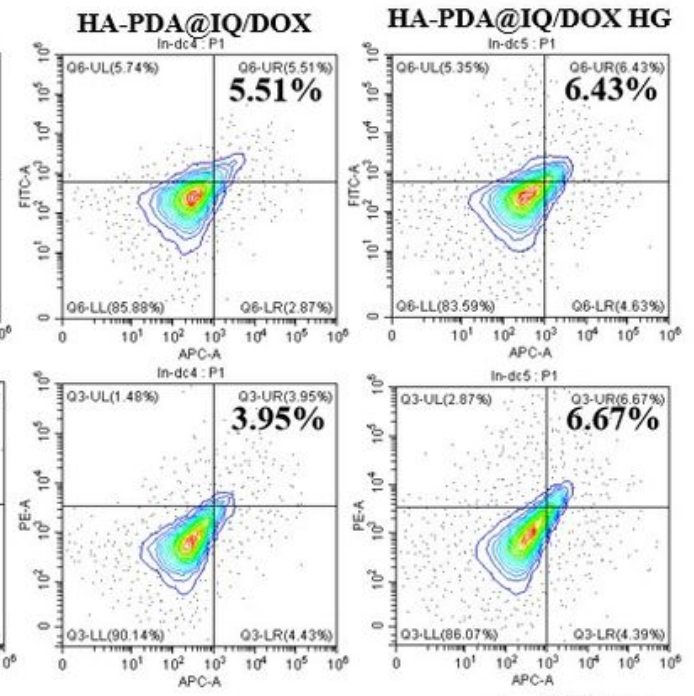

CD11c

HA-PDA@IQ

HA-PDA@IQ/DOX HA-PDA@IQ/DOX HG
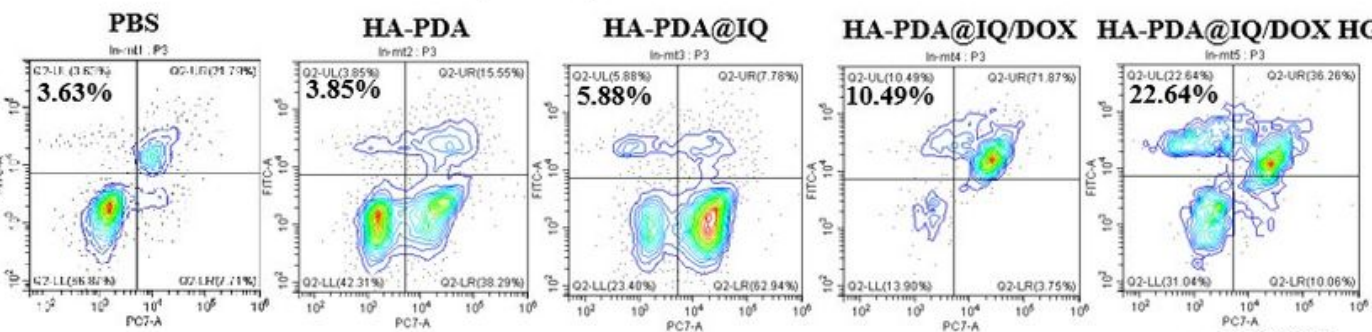

\section{LN ( $\left.\mathbf{T}_{\text {EM}}\right)$}
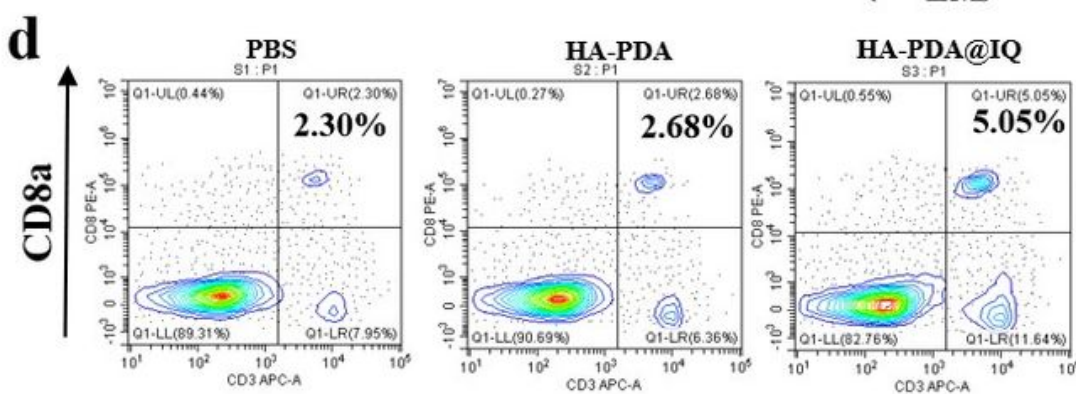

HA-PDA@IQ/DOX
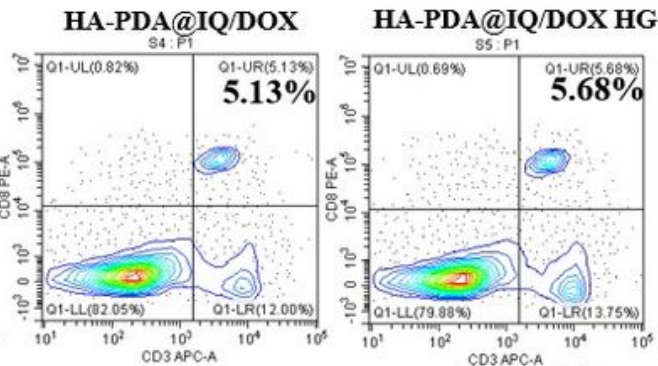

Spleens ( $C D 8+T$ cells)

CD62L

\section{Figure 7}

Immune responses assessment in vivo. (a) Scheme and timeline of the experimental design to evaluate the in vivo immune responses triggered by HA-PDA@IQ/DOX HG treatment. (b) Representative flow cytometry plots of CD80+ or CD86+ among CD11c+ DCs extracted from the inguinal lymph nodes (LNs). (c) Representative flow cytometry plots illustrated memory $T$ cell in inguinal lymph nodes (LNs). (d) Representative flow cytometry plots of CD3+CD8a+T cells in spleen. 
a
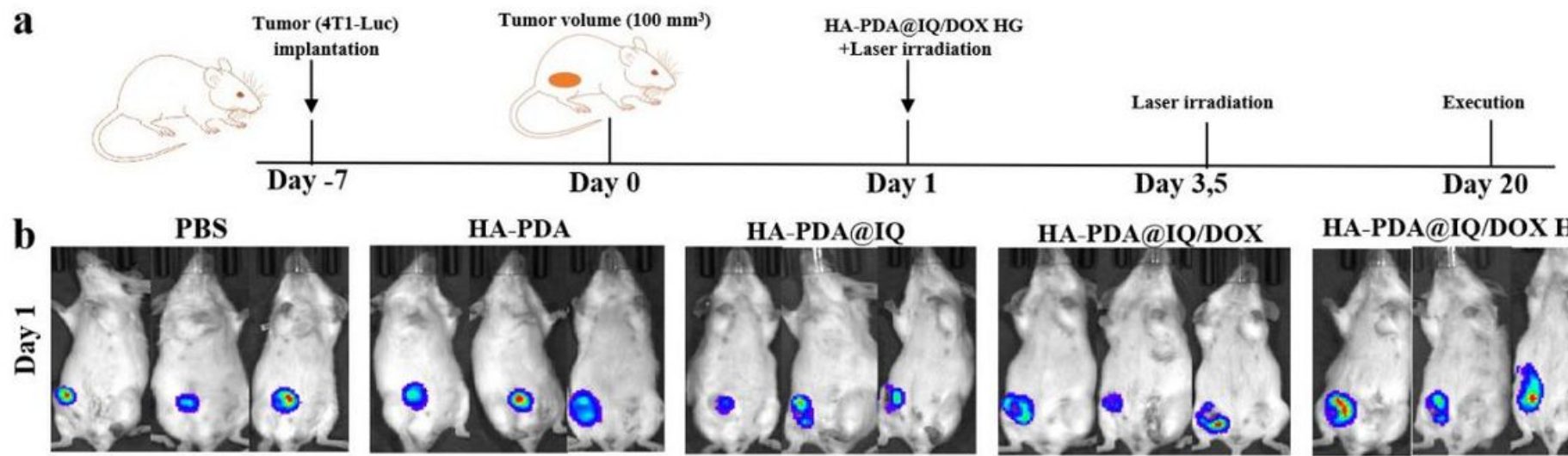

HA-PDA@IQ/DOX HG
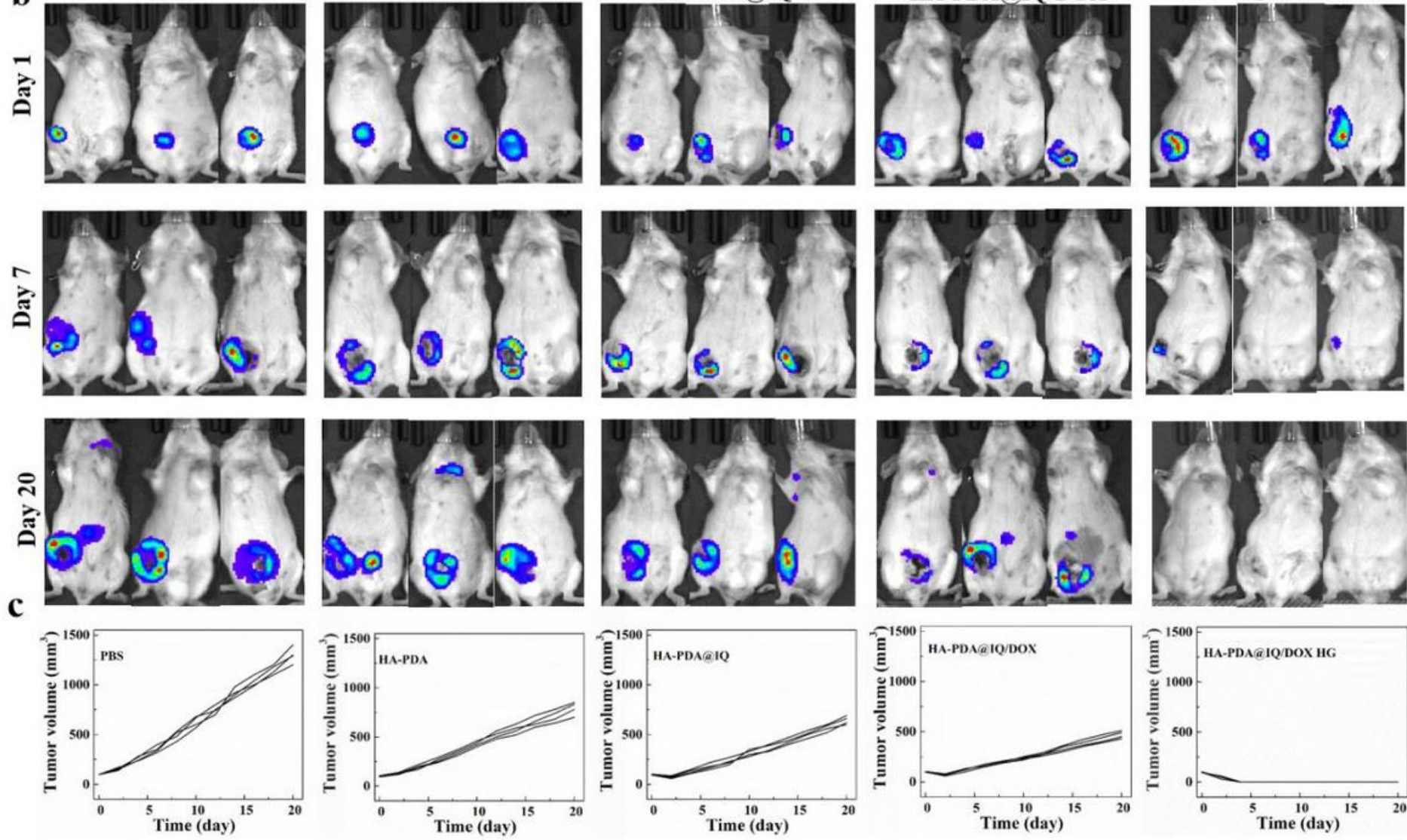

Figure 8

(a) Schematic diagram of experimental design for tumor therapy in vivo. (b) Luminescence imaging of 4T1-bearing mice and (c) corresponding individual tumor growth curves in different treatment until day 20 after treatment. 

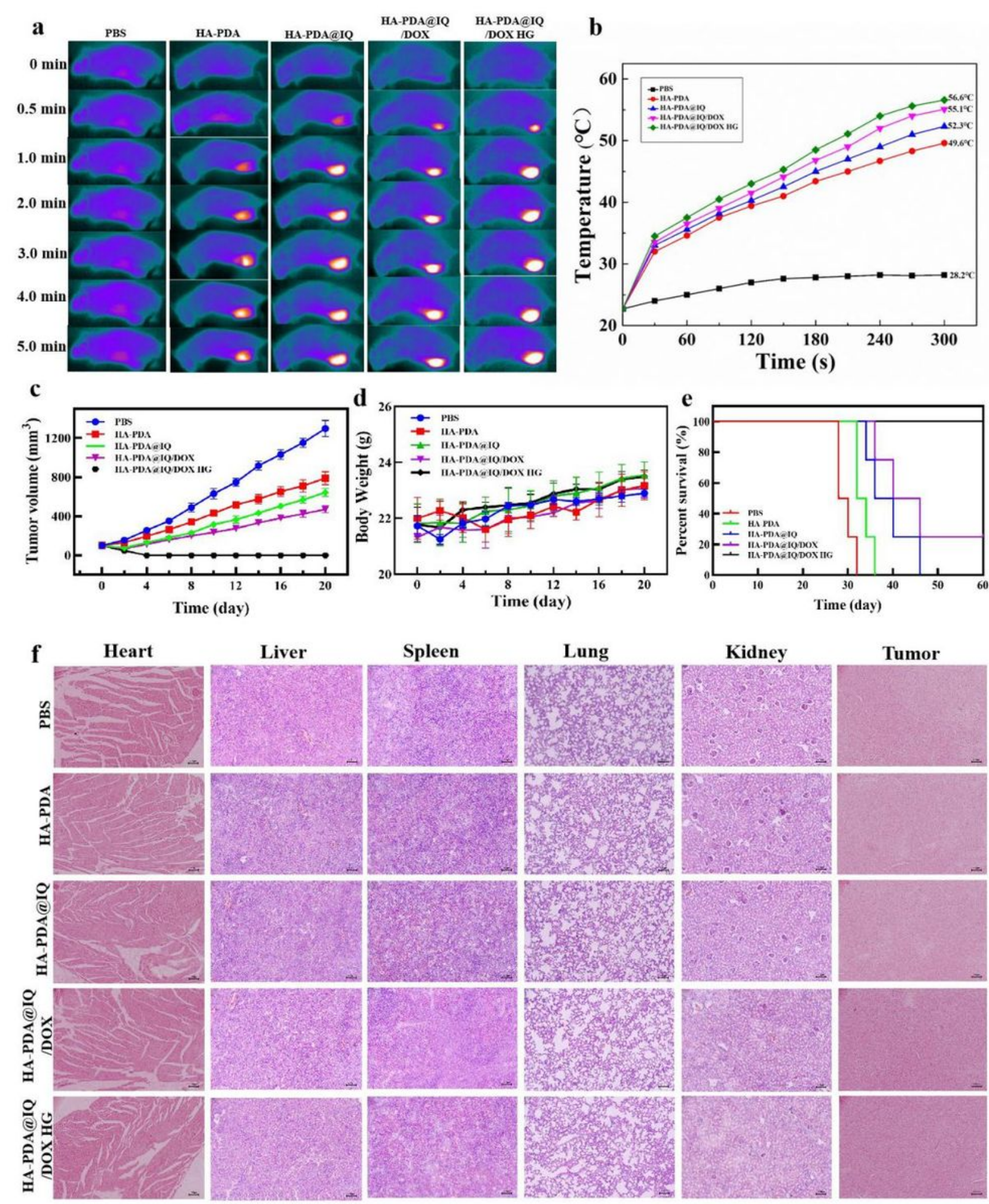

\section{Figure 9}

Characterization of HA-PDA@IQ/DOX HG mediated PTA using the 4T1 subcutaneous tumor-bearing mice model. (a) Infrared thermal images of mice at varied time points of treatment under different conditions using $808 \mathrm{~nm}$ laser irradiation. (b) Corresponding temperature change curves at the breast tumor sites of mice in different groups upon laser irradiation. (c) Tumor volume change curves, (d) the body-weight 
change, and (e) the survival curves of mice in the different groups. (f) H\&E-stained images of the major organs in the different groups.

\section{Supplementary Files}

This is a list of supplementary files associated with this preprint. Click to download.

- GraphicalAbstract.docx 